\title{
Galactic fountains and their connection with high and intermediate velocity clouds ${ }^{\star}$
}

\author{
E. Spitoni ${ }^{1}$, S. Recchi ${ }^{2}$, and F. Matteucci ${ }^{1,2}$ \\ 1 Dipartimento di Astronomia, Università di Trieste, via G.B. Tiepolo 11, 34131, Italy \\ e-mail: spitoni@oats.inaf.it \\ 2 INAF Osservatorio Astronomico di Trieste, via G.B. Tiepolo 11, 34131, Italy
}

Received 16 January 2008 / Accepted 17 March 2008

\section{ABSTRACT}

\begin{abstract}
Context. Sequential supernova explosions create supershells which can break out a stratified medium, producing bipolar outflows. The gas of the supershells can fragment into clouds which eventually fall toward the disk producing so-called galactic fountains. Aims. The aim of this paper is to calculate the expansion law and chemical enrichment of a supershell powered by the energetic feedback of a typical Galactic OB association at various galactocentric radii. We study the orbits of the fragments created when the supershell breaks out and we compare their kinetic and chemical properties with the available observations of high - and intermediate - velocity clouds.

Methods. We use the Kompaneets (1960, Soviet Phys. Dokl., 5, 46) approximation for the evolution of the superbubble driven by sequential supernova explosions and we compute the abundances of oxygen and iron residing in the thin cold supershell. We assume that supershells are fragmented by Rayleigh-Taylor instabilities and we follow the orbit of the clouds either ballistically or by means of a hybrid model considering viscous interaction between the clouds and the extra-planar gas.

Results. Given the self-similarity of the solutions, clouds are always formed $\sim 448 \mathrm{pc}$ above the plane. If the initial metallicity is solar, the pollution from dying stars of the $\mathrm{OB}$ association has a negligible effect on the chemical composition of the clouds. The maximum height reached by the clouds above the plane seldom exceeds $2 \mathrm{kpc}$ and when averaging over different throwing angles, the landing coordinate differs from the throwing coordinate by $\sim 1 \mathrm{kpc}$ at most.

Conclusions. The range of heights and $[\mathrm{O} / \mathrm{Fe}]$ ratios spanned by our clouds suggest that the high velocity clouds cannot have a Galactic origin, whereas intermediate velocity clouds have kinematic properties similar to our modeled clouds but have overabundances observed for the $[\mathrm{O} / \mathrm{Fe}]$ ratios that can be reproduced only with initial metallicities that are too low compared to those of the Galaxy disk.
\end{abstract}

Key words. ISM: jets and outflows - ISM: clouds - Galaxy: disk - Galaxy: open clusters and associations: general

\section{Introduction}

Although significant progress has been made in the last few years in exploring the distribution and chemical composition of intermediate and high-velocity clouds (IVCs and HVCs, respectively) in the halo of the Milky Way, an overall unified model for their formation is still lacking. These gas clouds are concentrations of neutral hydrogen (HI) with radial velocities that are not consistent with a simple model of differential galactic rotation (Richter et al. 2001). The distinction between HVCs and IVCs is loosely based on the observed radial velocities of the clouds; IVCs have radial velocities with respect to the Local Standard of Rest (LSR) of $30 \mathrm{~km} \mathrm{~s}^{-1} \leqslant\left|V_{\mathrm{LSR}}\right| \leqslant 90 \mathrm{~km} \mathrm{~s}^{-1}$ while HVCs have typical velocities $\left|V_{\mathrm{LSR}}\right|>90 \mathrm{~km} \mathrm{~s}^{-1}$. Several studies (Lu et al. 1998; Wakker et al. 1999; Murphy et al. 2000; Bluhm et al. 2001; Sembach et al. 2001) reveal different chemical compositions for several of these clouds in different directions in the sky. Oort (1970) proposed that these clouds represent condensed gaseous relics from the formation phase of the Milky Way. This idea was later revived by Blitz et al. (1999), who suggested that HVCs represent the building blocks of galaxies in a hierarchical galaxy formation scenario. Since the Galaxy is surrounded

\footnotetext{
* Appendix A is only available in electronic form at http://www. aanda.org
}

by smaller satellite galaxies (e.g., the Magellanic Clouds), another explanation is that IVCs and HVCs are gaseous streams related to the merging and accretion of these satellites by the Milky Way. In this picture, HVCs would be the gaseous counterparts of the Milky Way circumgalactic stellar streams, which are believed to represent the relics of dwarf galaxies that have been accreted by the Milky Way (e.g., Ibata 1994). While all these models assume that HVCs are truly extragalactic objects which are about to merge with the Galaxy from outside, there are other scenarios that see the IVCs and HVCs as objects that have their origin in the disk of the Milky Way, e.g., as part of the so-called "galactic fountains". In the galactic fountain model (Shapiro \& Field 1976; Houck \& Bregman 1990), hot gas is ejected out of the Galactic disk by supernova ( $\mathrm{SN}$ ) explosions, and part of this gas falls back in the form of condensed neutral clouds which move at intermediate and high radial velocities. Whatever the origin of the Milky Way IVCs and HVCs is, it has become clear that they must play an important role in the evolution of our Galaxy. Two extremely important parameters to distinguish between the Galactic and extragalactic models of IVCs and HVCs are the distance and the chemical composition of these clouds. In case of the Galactic model the clouds ejected by SN explosions must have a velocity high enough to explain the observed distances and their chemical composition must be correlated with the abundances of the gas in the disk. 
In this paper we examine the scenario in which the origin of these clouds is given by SN explosions, as the Galactic fountain model prescribes. We present precise calculations about the physical and chemical properties of the swept up gas by sequential SN events, in the literature called a supershell. Once the clouds leave the stellar disk, they move through the extra-planar gas halo. Two extreme types of models have been considered here for the motion of the gas through the extra-planar gas: i) the ballistic and ii) the fluid homogeneous models. Numerous non-homogenous filamentary structures observed in the extraplanar halos (NGC 891, NGC 5775) suggest that the kinematics of these halos could be interpreted with ballistic models. Ballistic models describe the gas as an inhomogeneous collection of clouds, subject only to the gravitational potential of the galaxy: for example, in the galactic fountain model the ejected gas from SN events falls back ballistically (Bregman 1980). These models are able to explain the vertical motion of the cold and warm gas components observed in several spiral galaxies (e.g. Fraternali et al. 2004; Boomsma et al. 2005). However, Collins et al. (2002) tried a ballistic model to describe the extraplanar ionized gas of NGC 891 and found problems in reproducing the observed kinematics. The ballistic model predicts that clouds migrate radially outward as they cycle through the halo. The mass fluxes estimated from the models of NGC 891 and NGC 5775 imply that significant amounts of gas can be involved in these migrations. Such migrations could cause a redistribution of gas that could affect metallicity gradients as well as star formation properties. Such effects have been investigated by Charlton \& Salpeter (1989), for example, but extensive observations of the kinematic behavior of edge-on galaxies should yield important constraints on such redistribution. Bregman (1980) presented a model where the clouds ejected fall into the disk subject to the gravity of the Galaxy at the same coordinates where they were ejected. In this case the metallicity gradient of the Galaxy does not change.

Collins et al. (2002) suggested that the ballistic model problems could be solved by considering the presence of drag between disk and halo, such as through magnetic tension or viscous interactions between clouds. Barnabè et al. (2006) presented fluid stationary models able to reproduce the observed negative vertical gradient of the rotation velocity of the extraplanar gas. In these models the gas is described as a stationary rotational fluid. In the case of a single cloud, the solutions of this model predict the drag invoked by Collins et al. (2002) as ram pressure between cloud and fluid halo. This suggests that a correct description of the extra-planar gas dynamics may be found in hybrid ballistic-fluid stationary models. Recently, Fraternali \& Binney (2008) presented a new formulation for viscous cloudhalo interactions in the framework of cold gas accretion onto spiral galaxies in the local universe.

In this paper we will follow the clouds ejected by SN explosions in the Galaxy both in the purely ballistic model and in the hybrid one. The paper is organized as follows: in Sect. 2 we will describe our model, in Sect. 3 we will show our results concerning the chemical abundances of the supershells and the cloud orbits in the purely ballistic and in the hybrid model. In Sect. 4 we compare our results with observations of the HVC Complex $\mathrm{C}$ and the IVC VIArc. In Sect. 5 we report our conclusions.

\section{Description of the model}

Type II SNe usually occur in OB associations containing several dozen massive stars. Sequential SN explosions create a superbubble, whereas the swept up gas is concentrated in a thin cold shell called a supershell. The superbubble expansion in a stratified medium does not follow a spherical evolution and the Kompaneets (1960) approximation well describes the way in which the superbubble grows in the meridional Galaxy plane. After some time, a region of the supershell can fragment due to the occurrence of Rayleigh-Taylor instabilities (RTIs), therefore clouds of gas can form. Once it has left the stellar disk, the orbit of each cloud can be followed either ballistically or with a hybrid model considering viscous interaction between the cloud and the extra-planar gas.

\subsection{The Kompaneets approximation}

The physical parameters of the superbubble can be fairly well described by the Kompaneets (1960) model. The Kompaneets approximation assumes the following: uniform pressure within the superbubble, superbubble expansion in a direction normal to the local surface and an expansion speed implied by a strong shock (i.e., the internal pressure dominates the external pressure). Kompaneets found an analytic expression for the shape of the bubble during its expansion in an exponential atmosphere with density:

$\rho(z)=\rho_{0} \exp (-z / H)$,

where $\rho_{0}$ and $H$ are the disk density and height scale, respectively. We consider the shock expansion in cylindrical coordinates $(z, R)$. In the work of Kompaneets it was assumed that for $z<0$ the ISM was denser than the gas residing above the disk. Therefore, in this approximation, the bubble has an asymmetric evolution with respect to the plane of the galaxy. Kompaneets found analytic expressions for the top and bottom sites of the remnant. Referring to Fig. 1 we have:

$z_{L, H}=-2 H \ln \left(1 \mp \frac{y}{2 H}\right)$,

and

$b=2 H \arcsin \left(\frac{y}{2 H}\right)$.

The quantity $y$ is a transformed variable (with units of length) defined by:

$y=\int_{0}^{t} \sqrt{\frac{\gamma+1}{2} \frac{E_{\mathrm{th}}}{\rho_{0} \Omega}} \mathrm{d} t$

where $\Omega$ and $E_{\mathrm{th}}$ and $\gamma$ are the volume, the thermal energy and the ratio of specific heats for the superbubble, respectively. The thermal energy is calculated from the differential equation:

$\frac{\mathrm{d} E_{\mathrm{th}}}{\mathrm{d} t}=L_{0}-P \frac{\mathrm{d} \Omega}{\mathrm{d} t}$,

where $L_{0}$ is the wind luminosity and the pressure is given by the equation of state:

$P=(\gamma-1) E_{\mathrm{th}} / \Omega$.

In our work for the ISM $z$-profile we assume an exponential law also for $z<0$. Hence the superbubble evolves symmetrically with respect to the galactic plane resulting in a peanut-like shape, as suggested by Mac Low et al. (1988) and by TenorioTagle et al. (1999). The volume of the superbubble can be estimated by:

$\Omega=2 \cdot \frac{4}{3} \pi a^{2} b-\frac{4}{3} \pi\left|z_{\mathrm{H}}\right|^{3}$ 


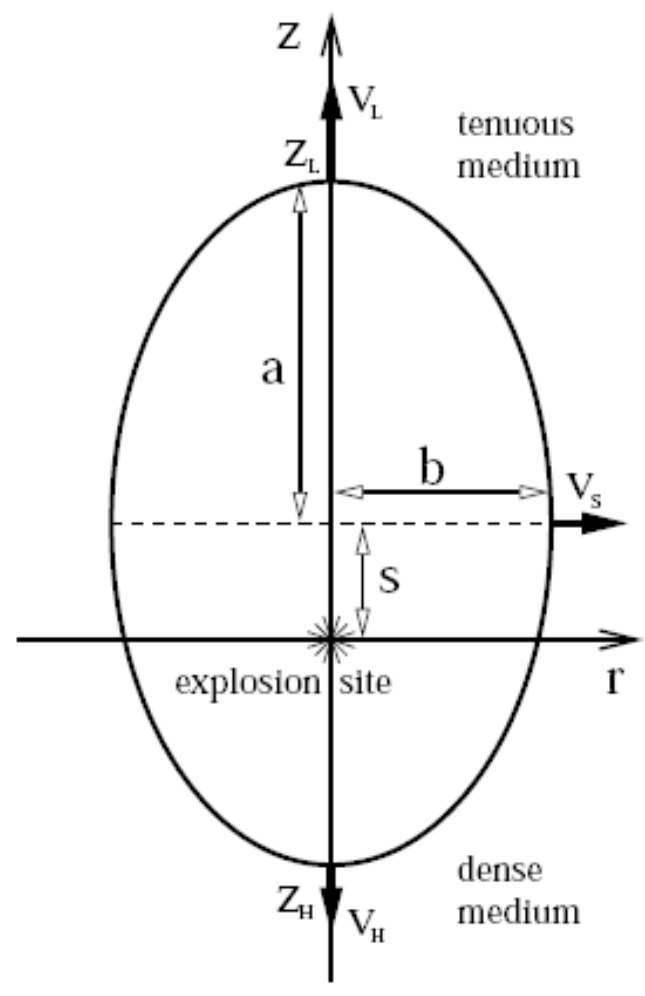

Fig. 1. The superbubble shape (see Kompaneets 1960) under the assumption that the density below the plane is constant (see text). Some physical quantities are reported: the height of the superbubble in the exponential atmosphere $\left(Z_{\mathrm{L}}\right)$ and below tha plane $\left(Z_{\mathrm{H}}\right)$, and the semiminor axis $(b)$. Figure taken from Kompaneets (1960).

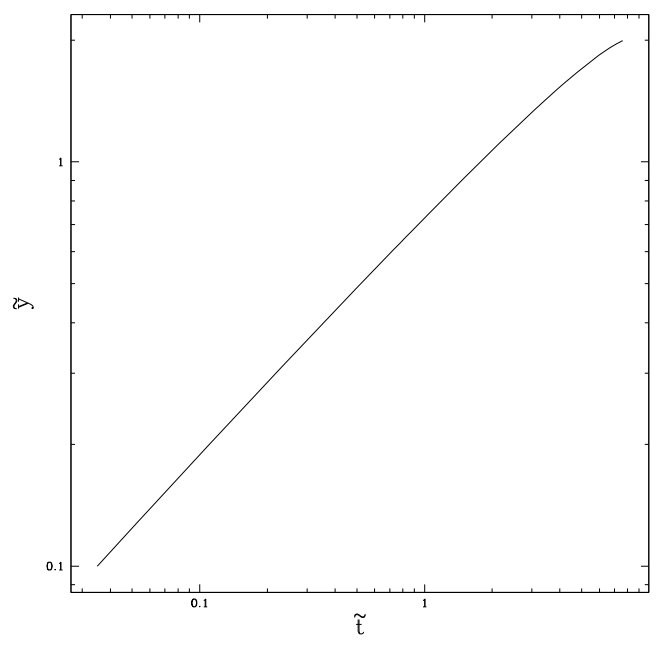

Fig. 2. Evolution in dimensionless space of the quantity $\tilde{y}$ (Eq. (4)) as a function of $\tilde{t} . \tilde{y}$ is related to the top of the superbubble $\left(Z_{\mathrm{L}}\right)$ evolution from Eq. (2). The dimensional time $t$ is given by: $t=\tilde{t} \times\left(\rho_{0} H^{5} / L_{0}\right)^{1 / 3}$.

where $a=\left(z_{\mathrm{L}}+\left|z_{\mathrm{H}}\right|\right) / 2$ is the semi-major axis. A numerical integration of Eqs. (4) and (5), with the help of Eqs. (6) and (7), yields $y(t)$ and $E_{\mathrm{th}}$ which implicitly give the time evolution of the superbubble. The solution depends on only three parameters: the scale height $H$, the density near the source $\rho_{0}$, and the wind luminosity $L_{0}$. Hence the problem is solved most naturally in a system of units determined by these three parameters. The unit of length is then $H_{0}$, the unit of mass is $\rho_{0} H^{3}$, and the unit of time is $\left(\rho_{0} H^{5} / L_{0}\right)^{1 / 3}$. We obtain the dimensionless solution by

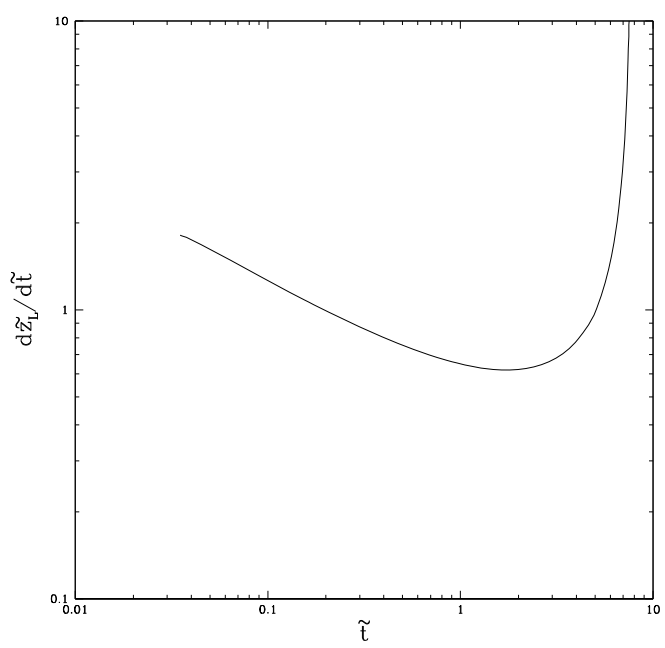

Fig. 3. Evolution in dimensionless space of the velocity $\mathrm{d} \tilde{\mathrm{L}} / \mathrm{d} \tilde{t}$ as function of $\tilde{t}$. The shell starts accelerating at $\tilde{t}_{\mathrm{a}}=1.72$.

integrating the dimensionless form of Eqs. (4) and (5). In Figs. 2 and 3 we report the dimensionless evolution of $y$ and the velocity $\mathrm{d} z_{\mathrm{L}} / \mathrm{d} t$. The dimensionless analysis predicts that the superbubble begins to accelerate at (see Fig. 3):

$\tilde{t_{\mathrm{a}}}=\frac{t_{\mathrm{a}}}{\left(\rho_{0} H^{5} / L_{0}\right)^{1 / 3}}=1.72$

namely when $\mathrm{d} z_{\mathrm{L}} / \mathrm{d} t$ is at a minimum. The simulated superbubbles, characterized by different values of $\rho_{0}, H, L_{0}$, reach the accelerated phase at different times, as shown in Eq. (8), but they have the same shape and volume. This is a direct consequence of the self-similar nature of the Kompaneets solutions. Using the scale height $H=141$ pc (see Sect. 2.3) we obtain for the height of the supeshell and for the minor semiaxis the values $z_{\mathrm{L}}=191 \mathrm{pc}$ and $b=145 \mathrm{pc}$. From Fig. 3 we note that, after the beginning of the accelerated phase, the velocity remains roughly constant for $\sim 2 \tilde{t}$. In this interval of time the acceleration is negligible. Therefore we assume that the RTIs begin to grow when the velocity is increased by a factor of $10 \%$, because from that moment on we can assume that the acceleration is not negligible. The choice of this percentage is arbitrary but in this way our results are consistent with the work of Mac Low \& McCray (1988). We have also tested velocity variations of $5 \%$ and $20 \%$ and the results are not very different to the ones presented in this paper. In our model we assume that due to the RTIs, the supershell fragments and we consider each fragment as a cloud with an initial velocity given by the supershell velocity at the moment of the fragmentation. RTIs arise when the supershell accelerates since at that moment, the effective gravity of the supershell exceeds that of the tenuous superbubble. From Tenorio-Tagle et al. (1987) we have an estimate of the time of growth of these highly irregular structures:

$t_{R-T}=10^{5} R_{40}^{2} \sqrt{\frac{\lambda_{10}}{E_{51}\left(t_{\mathrm{a}}+t_{\Delta v}\right)} n_{0}} \mathrm{yr}$

where $\lambda_{10}$ and $R_{40}$ are perturbation wavelength and remnant radius measured in units of 10 and $40 \mathrm{pc}$, respectively, $E_{51}$ is the energy measured in units of $10^{51} \mathrm{erg}$ and $t_{\Delta v}$ is the time necessary to reach an appreciable acceleration. At the time when RTIs start growing, the supershell has reached $z_{\mathrm{L}}=312$ and $b=228$. For 
simplicity we assume the formation of a single cloud that corresponds to the maximum perturbation wavelength for the thin shell, roughly given by the supershell thickness. In the case of adiabatic expansion of a spherical thin shell, the thickness $\delta$ is given by $\delta=R / 12$, where $R$ is the radius of the shell. We assume that $\delta$ is the maximum perturbation wavelength and we make the approximation that $R=z_{\mathrm{L}}$, therefore, $\delta=\lambda=26 \mathrm{pc}$. If we define $\tilde{t}_{R-T}$ as:

$\tilde{t}_{R-T}=\frac{t_{R-T}}{\left(\rho_{0} H^{5} / L_{0}\right)^{1 / 3}}$

we obtain the total adimensional time necessary for the growth of instabilities and for the fragmentation of the bubble:

$\tilde{t}_{\text {final }}=\tilde{t}_{\mathrm{a}}+\tilde{t}_{\Delta v}+\tilde{t}_{R-T}=4.37$.

Considering different values of the parameters $\rho_{0}, H, L_{0}$, we obtain different times at which the cloud could leave the disk:

$t_{\text {final }}=4.37\left(\rho_{0} H^{5} / L_{0}\right)^{1 / 3}$.

Since the internal pressure dominates the external pressure, the expansion speed is that given by the Hugoniot condition for a strong shock:

$v_{n}=\sqrt{\frac{\gamma+1}{2} \frac{P(t)}{\rho(z)}}$.

\subsection{Abundances of $\mathrm{Fe}$ and $\mathrm{O}$ in the superbubble}

In this section we compute the total amounts of $\mathrm{O}$ and $\mathrm{Fe}$ that reside in the supershell at the time $t_{\text {final }}$ (see Eq. (12)) in which we suppose the fragmented shell can leave the stellar disk of the Galaxy. We assume that all the stars with masses greater than $8 M_{\odot}$ explode as type II SNe and we adopt the following main sequence lifetimes (Matteucci \& Greggio 1986):

$\tau_{m}=1.2 m^{-1.85}+0.003 \mathrm{Gyr}$.

In our model, the upper mass limit of the OB association is assumed to be $40 M_{\odot}$. The lifetime for a $40 M_{\odot}$ association is, from Eq. (14), $\tau_{40} \simeq 4.30 \times 10^{6} \mathrm{yr}$.

For any parameter choice, at the time at which the shell fragments and leaves the disk, the smaller mass stars in the OB association are still active. Using Eq. (14) we are able to estimate the lower mass limit for a SN event corresponding to the time at which the cloud forms. The time at which the cloud is formed is given by $t_{\text {final }}$ plus the lifetime of the largest considered mass, namely:

$\tau_{\text {lim }}=t_{\text {final }}+\tau_{40}$.

The mass of metals (in our model we consider only $\mathrm{O}$ and $\mathrm{Fe}$ ) ejected by the range of SNe considered is given by:

$M_{\mathrm{el}_{\star}}=\int_{M_{\mathrm{inf}}}^{40} m_{\mathrm{el}}(m) \phi(m) \mathrm{d} m$,

where $\phi(m)$ is the initial mass function (IMF) assumed to be the Salpeter (1955) one, namely: $\phi(m)=A m^{-(2.35)}$. A is the normalization constant, given by:

$\int_{8}^{40} \phi(m) \mathrm{d} m=A \int_{8}^{40} m^{-2.35} \mathrm{~d} m=\mathrm{SNe}$,

where $\mathrm{SNe}$ is the assumed number of $\mathrm{SNe}$ in the $\mathrm{OB}$ association. In Eq. (16) the term $m_{\mathrm{el}}(m)$ is the ejected mass of the el element considered $(\mathrm{O}$ or $\mathrm{Fe}$ ) by the star of initial mass $m$ (Woosley \& Weaver 1995). We consider the stellar yields at four different metallicities: solar metallicity $Z_{\odot}, 0.1 Z_{\odot}, 0.01 Z_{\odot}, 10^{-4} Z_{\odot}$. We assume that the ISM in the disk has the same metallicity as the OB association. For the solar abundances (by mass) we use the Anders \& Grevesse (1989) values:

$\mathrm{O}_{16_{\odot}}=9.59 \times 10^{-3}, \mathrm{Fe}_{56_{\odot}}=1.17 \times 10^{-3}$.

We use this set of solar abundances to be consistent with the yields of Woosley \& Weaver (1995) which refer to Anders \& Grevesse (1989) abundances. However Asplund et al. (2005) recomputed the $\mathrm{O}$ solar abundance and deduced a $\log (\mathrm{O} / \mathrm{Fe})_{\odot}$ lower by $\sim 0.24$ dex than the Anders \& Grevesse (1989) one. The total amount $M_{\text {shell-el }}$ of the mass of the element which resides in the thin shell of the superbubble is given by the equation:

$M_{\text {shell-el }}=M_{\text {shell }} \cdot Z+M_{\mathrm{el}_{\star}}$,

where $Z$ is the initial metallicity of the $\mathrm{OB}$ association (and consequently of the disk ISM). We adopt the extreme approximation that the total amount of the ejected metals from SNe ends up in the shell. $M_{\text {shell }}$ is the mass of the ISM swept up into the thin shell and is given by:

$M_{\text {shell }}(z>0)=\pi \rho_{0} b^{2} \int_{0}^{z_{\mathrm{L}}} \mathrm{e}^{-z / H}\left(1-\left(\frac{z-a+\left|z_{\mathrm{H}}\right|}{a}\right)^{2}\right) \mathrm{d} z$.

We underline that for the Fe we use only solar yields for the whole range of metallicities (Chiappini et al. 2001).

\subsection{Galaxy model}

The potential well of the Galaxy is assumed to be the sum of three components: a dark matter halo, a bulge and a disk. The dark matter halo gravitational potential is assumed to follow the Navarro et al. (1996) profile:

$\Phi_{\mathrm{dm}}(r)=-4 \pi r_{\mathrm{dm}, 0}^{2} \rho_{\mathrm{dm}, 0} \frac{\ln (1+x)}{x}$,

where $\rho_{\mathrm{dm}, 0}$ is a reference density, $r_{\mathrm{dm}, 0}$ is the scale radius, $x=$ $r / r_{\mathrm{dm}, 0}$ and $r$ is the spherical radius. The halo is truncated at a radius $r_{\mathrm{dm}, t}$ beyond which its potential follows the $1 / \mathrm{r}$ profile. The bulge gravitational potential is given by (Hernquist 1990):

$\Phi_{\mathrm{b}}(r)=-\frac{G M_{\mathrm{b}}}{r_{\mathrm{b}, 0}+r}$

where $r_{\mathrm{b}, 0}$ is the scale radius and $M_{\mathrm{b}}$ is the bulge mass. For the disk potential we have chosen the axisymmetrical Miyamoto \& Nagai (1975) model, which in cylindrical coordinates $(R, z)$ can be written as:

$\Phi_{M}(R, z)=-\frac{G M_{d}}{\sqrt{R^{2}+\left(a+\sqrt{z^{2}+b^{2}}\right)^{2}}} \cdot$

Table 1 gives the values of all the parameters concerning the Galaxy model. For the interstellar medium (ISM) $z$-density profile we used Eq. (1) where: $\rho_{0}=n_{0} \mu m_{\mathrm{p}}$ is the density in the disk plane; $m_{\mathrm{p}}$ is the proton mass and $\mu$ is mean molecular weight for the disk (assumed to be 0.61). For the value of $\mathrm{H}$ we adopted the vertical distributions of the various interstellar components (molecular, cold HI, warm HIa, warm HIb, HII regions, and diffuse HII) in the solar neighborhood as reported by Cox (2005). We obtained:

$H=\frac{1}{\rho_{0}} \int_{0}^{\infty} \sum_{i=1}^{6} \rho_{i}(z) \mathrm{d} z \simeq 141 \mathrm{pc}$. 
Table 1. Galactic parameters.

\begin{tabular}{cccccccc}
\hline \hline $\begin{array}{c}\rho_{\mathrm{dm}, 0} \\
10^{-24} \mathrm{~g} \mathrm{~cm}^{-3}\end{array}$ & $\begin{array}{c}M_{\mathrm{b}} \\
10^{10} M_{\odot}\end{array}$ & $\begin{array}{c}M_{\mathrm{d}} \\
10^{10} M_{\odot}\end{array}$ & $\begin{array}{c}r_{\mathrm{dm}, 0} \\
\mathrm{kpc}\end{array}$ & $\begin{array}{c}r_{\mathrm{dm}, t} \\
\mathrm{kpc}\end{array}$ & $\begin{array}{c}r_{\mathrm{b}, 0} \\
\mathrm{kpc}\end{array}$ & $\begin{array}{c}a \\
\mathrm{kpc}\end{array}$ & $\begin{array}{c}b \\
\mathrm{kpc}\end{array}$ \\
\hline 0.29 & 3.5 & 7.69 & 30.8 & 347.7 & 0.8 & 8.45 & 0.26 \\
\hline
\end{tabular}

The ISM density profile along the radius $R$ of the Galaxy is taken from Wolfire et al. (2003). In our model we consider the presence of an extra-planar gas halo. This halo can be described by means of the perfect gas law:

$P=\frac{\rho k T}{\mu m_{\mathrm{p}}}$

For simplicity we assume an isothermal and self-graviting distribution, hence introducing the parameter:

$\beta_{0}=\frac{k T_{0}}{\mu m_{\mathrm{p}}}$,

by means of which eq. (25) can be written as:

$P=\beta_{0} \rho$.

Using the hydrostatic equilibrium equation with the assumption of a static halo of gas we have:

$\rho(R, z)=\rho_{\mathrm{o}} \mathrm{e}^{\left(-\Phi_{\mathrm{tot}}+\Phi_{0}\right) / \beta_{0}}$,

where $\Phi_{\text {tot }}=\Phi_{\mathrm{dm}}+\Phi_{\mathrm{b}}+\Phi_{\mathrm{M}}$ and $\Phi_{0}$ is the value of $\Phi_{\text {tot }}$ calculated at the galactic center. The temperature $T_{\mathrm{o}}$ has been chosen following the relation:

$\frac{3}{2} k T_{\mathrm{o}}=\frac{3 m_{\mathrm{p}} \mu v_{\mathrm{c} 8}^{2}}{2}$,

where $v_{\mathrm{c} 8}$ is the circular velocity in the plane of Galaxy in the solar neighborhood and we obtain that:

$T_{0}=3.7 \times 10^{6} \mathrm{~K}$

for the chosen parameters.

\subsection{Galactic fountains in a ballistic model}

Once the top of the supershell reaches the height above the galactic plane related to the time $t_{\text {final }}$ (see Eq. (12)), the thin shell can leave the stellar disk and move towards the extra-planar gas. Ballistic models describe the gas as an inhomogeneous collection of clouds, subject only to the gravitational potential of the Galaxy. The fragments of the shell have different initial velocity modulus $\left\|\boldsymbol{v}_{\mathrm{o}}\right\|=v_{n}$ and masses depending on the number of $\mathrm{SNe}$ in the $\mathrm{OB}$ association and the initial throwing radial coordinate. We have chosen for our simulation Cartesian coordinates with versor $\left(\hat{\boldsymbol{e}}_{x}, \hat{\boldsymbol{e}}_{y}, \hat{\boldsymbol{e}}_{z}\right)$. Given a generic point in this space $(x, y, z)$, it corresponds in the meridional plane of the Galaxy to $(R, z)=\left(\sqrt{x^{2}+y^{2}}, z\right)$, where $R$ is the radial coordinate. Since the $\left\|\boldsymbol{v}_{\mathrm{o}}\right\|$ velocities are relative to the local standard of rest for the throwing radial coordinate $R_{0}$, in the inertial reference frame of the simulation in Cartesian coordinates:

$\boldsymbol{v}_{\text {initial }}\left(R_{0}\right)=\boldsymbol{v}_{\mathrm{o}}+v_{\mathrm{c}}\left(R_{\mathrm{o}}, 0\right) \hat{\boldsymbol{e}}_{y}$.

For each choice of $\left\|\boldsymbol{v}_{\mathrm{o}}\right\|$ we consider several throwing directions to take into account the possibility that the breakup of a single supershell produces several fragments, each one with its own orbit. Given a throwing direction, a generic vector $\boldsymbol{v}_{0}$ has components along $\hat{\boldsymbol{e}}_{x}, \hat{\boldsymbol{e}}_{z}$ and $\hat{\boldsymbol{e}}_{y}$ with respect to our inertial frame:

$\left\{\begin{array}{l}v_{z_{\text {init }}}=v_{n} \cos \gamma \\ v_{x_{\text {init }}}=v_{n} \sin \gamma \cos \beta \\ v_{y_{\text {init }}}=v_{n} \sin \gamma \sin \beta+v_{\mathrm{c}}\left(R_{0}, 0\right) .\end{array}\right.$

The parameters $\gamma$ and $\beta$ vary in order to recreate, in the local standard of rest, a symmetrical fountain of jets:

$\beta_{i}=\frac{i \pi}{4}$ with $i=0,1, \ldots 7$

and

$\gamma_{j}=\frac{\pi}{2}-\frac{\pi}{1+j}$ with $j=2,3, \ldots 5$.

We consider also the case in which $\boldsymbol{v}_{0}$ has only a component along $\hat{\boldsymbol{e}}_{z}$. Thus, for each velocity modulus $v_{n}$ we calculate a fountain composed of 33 jets.

\subsection{Beyond the ballistic model: hybrid ballistic-fluid stationary model}

In this paper we want to provide some hydrodynamical modifications of previous ballistic models. In the motion equations for a single cloud we insert a Stokes term in order also to consider the viscous interactions between cloud and extra-planar gas. We introduce the drag time $t_{\mathrm{drag}}$, i.e the time required for an HI cloud to corotate with a homogeneous gas halo (Barnabè et al. 2005):

$t_{\mathrm{drag}}=\frac{8}{3 C_{\mathrm{D}}} \frac{r_{\mathrm{c}}}{v_{\mathrm{rel}}} \chi$

where $C_{\mathrm{D}} \simeq 1$ is a numerical coefficient, $r_{\mathrm{c}}$ is the radius of a typical HI cloud, $v_{\text {rel }}$ is the modulus of the relative velocity between the cloud and the homogeneous extra-planar gas, and $\chi \equiv \rho_{\mathrm{c}} / \rho$ is the ratio between the cloud and the medium densities. $t_{\text {drag }}$ was estimated assuming pressure equilibrium between cold and hot components, from which $\chi=T / T_{\mathrm{c}} \approx 3000$. A fiducial value of the relative velocity is obtained by assuming $v_{\text {rel }} \approx 2 \times 10^{7} \mathrm{~cm} \mathrm{~s}^{-1}$, while the cloud radius $r_{\mathrm{c}}$ is estimated as:

$4 \pi r_{\mathrm{c}}^{3} \rho_{\mathrm{c}} / 3=10^{5} M_{\odot} \tilde{M}_{5}$,

where $\tilde{M}_{5}$ is the cloud mass in units of $10^{5} M_{\odot}$, thus following the work of Barnabè et al. (2005) one obtains:

$t_{\text {drag }} \approx 2.7 \times 10^{8}\left(\frac{\tilde{M}_{5}}{n_{\mathrm{p}}}\right)^{1 / 3} \mathrm{yr}$,

where $n_{\mathrm{p}}$ is the numerical density of the extra-planar gas with the density profile described by Eq. (28). We note that a viscosity term could not be directly inserted in a Lagrangian formulation, thus we must write the explicit drag term to add in the motion equations. The drag term can be derived from the brake force estimate as:

$\boldsymbol{a}_{\mathrm{drag}} \equiv-\frac{\boldsymbol{v}-\boldsymbol{v}_{g}}{t_{\mathrm{drag}}}$

where $v$ is the velocity of the cloud whereas $v_{\mathrm{g}}$ is the velocity for the extra-planar gas that we assumed equal to zero. 
Table 2. Cloud formation times and cloud velocities for $R_{0}=4 \mathrm{kpc}$.

\begin{tabular}{ccc}
\hline \hline SNe & $\begin{array}{c}t_{\text {final }} \\
{[\mathrm{yr}]}\end{array}$ & $\begin{array}{c}v_{\mathrm{n}} \\
{\left[\mathrm{km} \mathrm{s}^{-1}\right]}\end{array}$ \\
\hline 10 & $2.29 \times 10^{7}$ & 23 \\
50 & $1.34 \times 10^{7}$ & 39 \\
100 & $1.06 \times 10^{7}$ & 49 \\
500 & $6.21 \times 10^{6}$ & 83 \\
\hline
\end{tabular}

Table 3. Same as Table 2 but for $R_{0}=8 \mathrm{kpc}$.

\begin{tabular}{ccc}
\hline \hline SNe & $\begin{array}{c}t_{\text {final }} \\
{[\mathrm{yr}]}\end{array}$ & $\begin{array}{c}v_{\mathrm{n}} \\
{\left[\mathrm{km} \mathrm{s}^{-1}\right]}\end{array}$ \\
\hline 10 & $1.90 \times 10^{7}$ & 27 \\
50 & $1.11 \times 10^{7}$ & 46 \\
100 & $8.84 \times 10^{6}$ & 58 \\
500 & $5.17 \times 10^{6}$ & 100 \\
\hline
\end{tabular}

Table 4. Same as Table 2 but for $R_{0}=12 \mathrm{kpc}$.

\begin{tabular}{ccc}
\hline \hline $\mathrm{SNe}$ & $\begin{array}{c}t_{\text {final }} \\
{[\mathrm{yr}]}\end{array}$ & $\begin{array}{c}v_{\mathrm{n}} \\
{\left[\mathrm{km} \mathrm{s}^{-1}\right]}\end{array}$ \\
\hline 10 & $1.67 \times 10^{7}$ & 31 \\
50 & $9.77 \times 10^{6}$ & 53 \\
100 & $7.74 \times 10^{6}$ & 67 \\
500 & $4.53 \times 10^{6}$ & 114 \\
\hline
\end{tabular}

\section{Our results}

\subsection{Chemical composition of the clouds}

In our models we vary the number of SNeII in the OB association $(\mathrm{SNe})$ and the throwing radial coordinate $\left(R_{0}\right)$. We consider four possible OB associations containing 10, 50, 100, $500 \mathrm{SNe}$ respectively. Assuming an explosion energy of $10^{51} \mathrm{erg}$, the luminosities $L_{0}$ of these OB associations are $10^{37}, 5 \times 10^{37}, 10^{38}$ and $5 \times 10^{38} \mathrm{ergs}^{-1}$ respectively. These numbers of massive stars in $\mathrm{OB}$ associations are consistent with observations (de Zeeuw et al. 1999). We simulate galactic fountains with 3 different throwing radial coordinates $R_{0}: 4,8,12 \mathrm{kpc}$. Given the assumed Galaxy model, $R_{0}$ defines the disk density $\rho_{0}$, whereas the scale height is constant (see Sect. 2.3). In Tables 2-4 we summarize the results for fragmentation times and the velocities of the superbubbles in the direction perpendicular to the galactic plane at those times for $4 \mathrm{kpc}, 8 \mathrm{kpc}$ and $12 \mathrm{kpc}$.

For all the values of SNe and $R_{0}$ considered at the time $t_{\text {final }}$ at which clouds are thrown out of the disk, the supershell presents: $z_{\mathrm{L}}=448 \mathrm{pc}, z_{\mathrm{H}}=165 \mathrm{pc}$ and $b=259 \mathrm{pc}$. Our results are in agreement with the work of Mac Low \& McCray (1988): instabilities, for roughly the same luminosity range, become important at $3 H$ height scale. The total mass of gas swept up by the SN shock wave for positive z-coordinates is given by Eq. (20).

Thus, we obtain that the masses of the ISM swept up into the thin shell for $R_{0}=4,8,12 \mathrm{kpc}$, respectively are:

$$
\begin{aligned}
& M_{4}(z>0)=10.07 \times 10^{5} M_{\odot}, \\
& M_{8}(z>0)=5.79 \times 10^{5} M_{\odot}, \\
& M_{12}(z>0)=3.89 \times 10^{5} M_{\odot} .
\end{aligned}
$$

All the results of the $\mathrm{O}$ and $\mathrm{Fe}$ abundances in the clouds ejected by sequential $\mathrm{SN}$ explosions as functions of $\mathrm{SNe}$ and $R_{0}$ are

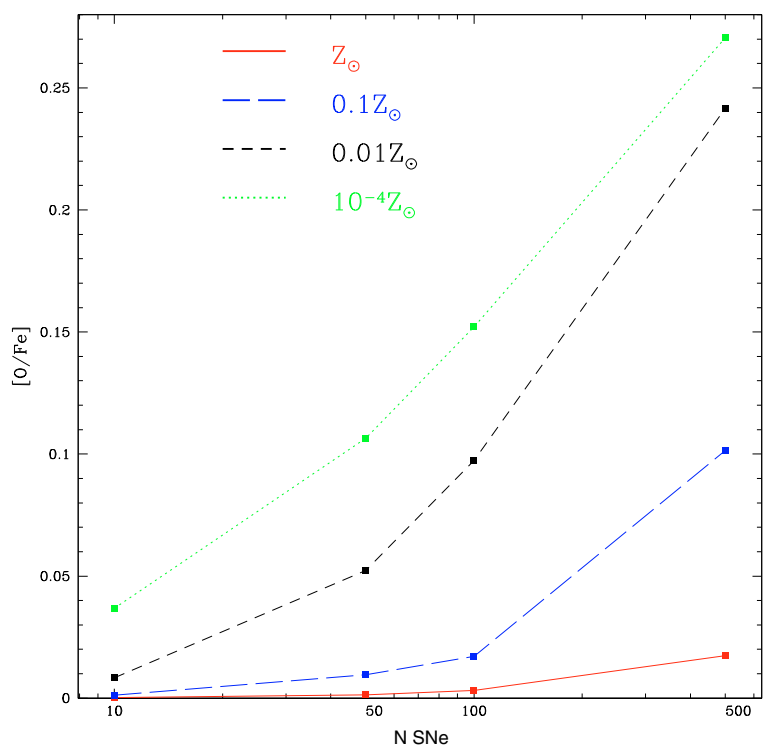

Fig. 4. $[\mathrm{O} / \mathrm{Fe}]$ ratios as functions of the number of $\mathrm{SNe}$ and of different disk gas metallicities in the case of $R_{0}=8 \mathrm{kpc}$ using stellar yields given by Woosley \& Weaver (1995).

reported in the online material. $M_{* \mathrm{Fe}_{56}}$ and $M_{* \mathrm{O}_{16}}$ are the total amounts of $\mathrm{Fe}_{56}$ and $\mathrm{O}_{16}$ in units of $M_{\odot}$, whereas $M_{\star \mathrm{ej}}$ is the total mass ejected by the $\mathrm{OB}$ association:

$M_{\star \mathrm{ej}}=\int_{M_{\mathrm{inf}}}^{40} m_{\mathrm{tot}}(m) \phi(m) \mathrm{d} m$,

where $m_{\text {tot }}(m)$ is the total mass ejected by a SN as a function of its initial mass and metallicity. $M_{\text {tot }}, X_{* \mathrm{Fe}_{56}}, X_{* \mathrm{O}_{16}}$ and $[\mathrm{O} / \mathrm{Fe}]$ are:

$M_{\text {tot }}=M_{\text {shell }}+M_{\star \mathrm{ej}}$

$X_{* \mathrm{Fe}_{56}}=\frac{M_{\text {ShellFe }_{56}}}{M_{\mathrm{tot}}}, X_{* \mathrm{O}_{16}}=\frac{M_{\mathrm{ShellO}_{16}}}{M_{\mathrm{tot}}}$.

$[\mathrm{O} / \mathrm{Fe}]=\log \left(\frac{M_{\text {Shell }_{16}}}{M_{\text {ShellFe }_{56}}}\right)-\log \left(\frac{\mathrm{O}_{16_{\odot}}}{\mathrm{Fe}_{56_{\odot}}}\right)$.

In Fig. 4 we show the predicted $[\mathrm{O} / \mathrm{Fe}]$ ratio as a function of the number of $\mathrm{SNe}$ and of the initial metallicity in the solar vicinity. In the meridional plane of the Galaxy the initial conditions are $(R, z)=(8 \mathrm{kpc}, 448 \mathrm{pc})$. We note that significant overabundances of $\mathrm{O}$ relative to $\mathrm{Fe}$ are found only in the case of a large number of $\mathrm{SNe}$ and low initial metallicity. In Fig. 5 we report the same quantities but using stellar yields given by Kobayashi et al. (2006). In Fig. 6 we show [O/Fe] ratios as functions of the number of SNe assuming solar metallicity but varying the initial throwing coordinate $R_{\mathrm{o}}$. We note that larger radial coordinates yield a larger $[\mathrm{O} / \mathrm{Fe}]$, because the amount of sweptup pristine gas is smaller (see Eq. (41)) and therefore the new $\alpha$ elements ejected by $\mathrm{SNe}$ are less diluted. In Fig. 7 we report the $[\mathrm{O} / \mathrm{Fe}]$ ratios varying the initial throwing coordinate $R_{0}$ and taking for the initial ISM metallicities the average observed values given by Andreievsky et al. (2002a-c, 2004) and Luck et al. (2003), as a function of galactocentric distance, by analyzing Galactic Cepheids (see Cescutti et al. 2006). Referring to Table 4 of the work of Cescutti et al. (2006), we find: $Z=1.65 \times Z_{\odot}$ for $R_{0}=4 \mathrm{kpc}$ and $Z=0.74 \times Z_{\odot}$ for $R_{0}=12 \mathrm{kpc}$. 


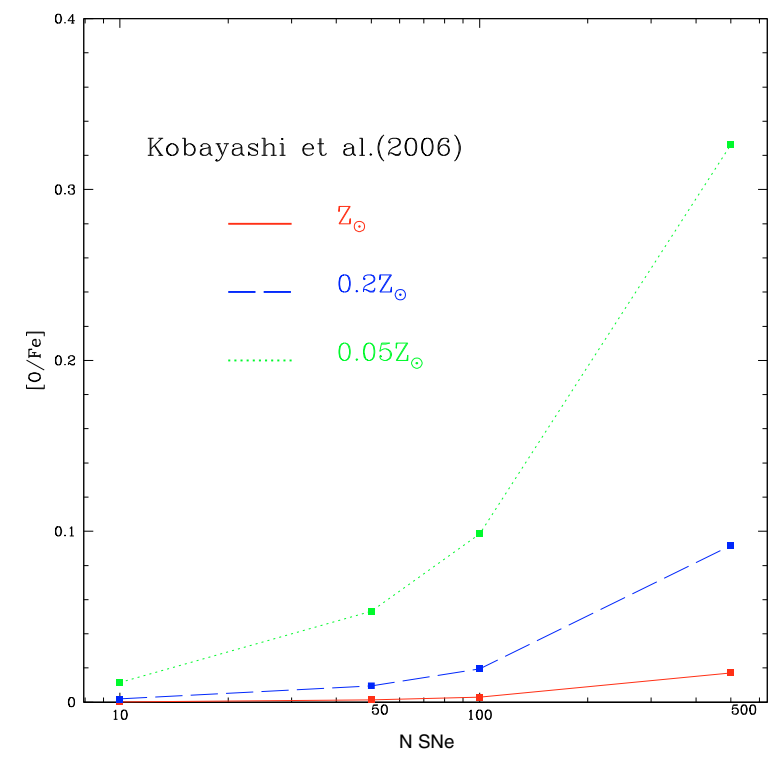

Fig. 5. As in Fig. 4 using stellar yields given by Kobayashi et al. (2006).

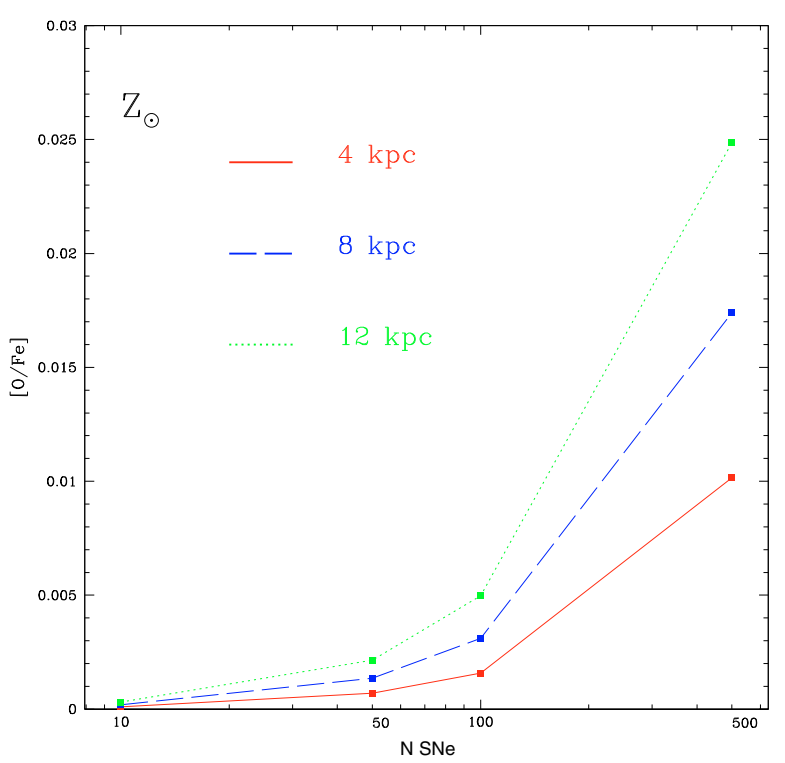

Fig. 6. $[\mathrm{O} / \mathrm{Fe}]$ ratios as functions of the number of $\mathrm{SNe}$ and the throwing radial coordinate in the case of solar metallicity using stellar yields given by Woosley \& Weaver (1995).

\subsection{Dynamics of the galactic fountains}

Our analysis focuses first on the study of solar neighborhood galactic fountains (i.e. with $R_{0}=8 \mathrm{kpc}$ ). In Fig. 8 we show our results concerning the orbits of the shell fragments once they leave the stellar disk in the purely ballistic model. The trajectories of the orbits of each galactic fountain are plotted in the meridional plane of the Galaxy $(R, z)$. Spatial initial conditions are the same for all cases considered: $R_{0}=8 \mathrm{kpc}$ and $z_{0}=448 \mathrm{pc}$, whereas the initial velocities depend on the number of SNe considered (Table 3). In Fig. 8, squares on the $R$ axis reported the average falling radial coordinate in the Galaxy plane. As we can see, the clouds are preferentially thrown outwards,

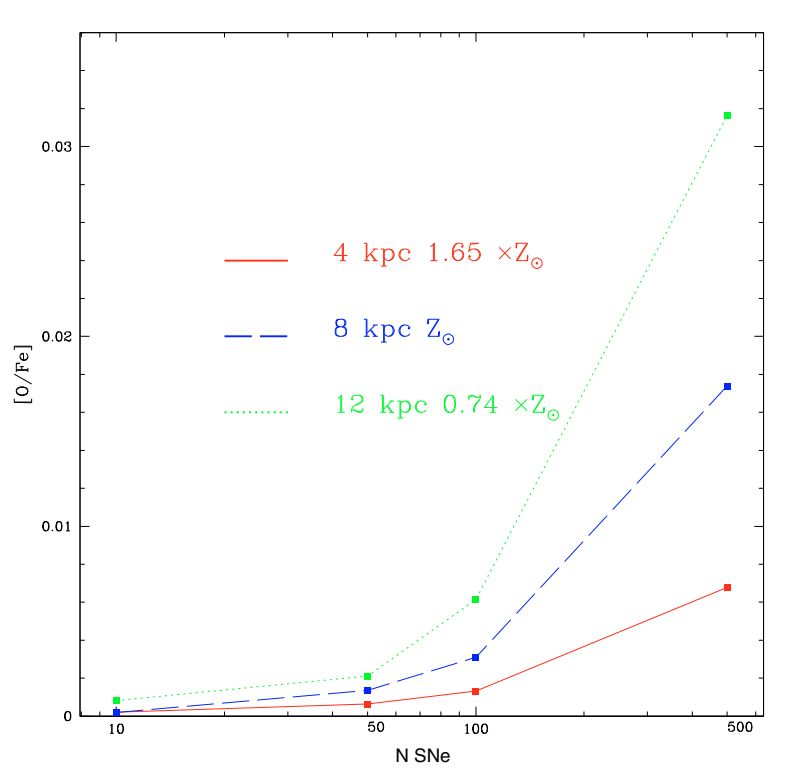

Fig. 7. $[\mathrm{O} / \mathrm{Fe}]$ ratios as functions of the number of $\mathrm{SNe}$ and the initial throwing radial coordinate, taking for the ISM metallicities the average observed values along the Galactic disk from Cepheids by Andreievsky et al. (2002a-c, 2004) and Luck et al. (2003) (see Cescutti et al. 2006).

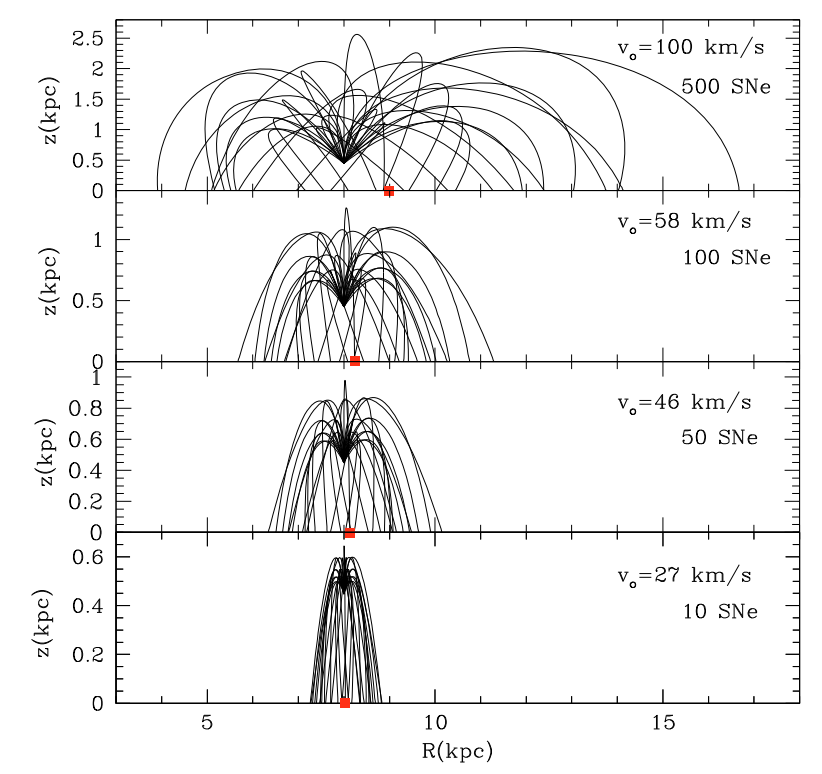

Fig. 8. Galactic fountains reported in the meridional plane in the purely ballistic model with the same spatial initial conditions: $(R, z)=(8 \mathrm{kpc}$, $448 \mathrm{pc})$. Squares on the $R$ axis are the average falling radial coordinate.

but their final average landing coordinates differ by $1 \mathrm{kpc}$ at most from the throwing coordinate. This result is consistent with the works of Bregman (1980), Fraternali \& Binney (2008) and Melioli et al. (2008). On the other hand, in their recent hydrosimulations for the Milky Way disk, Booth \& Theuns (2007) found that clouds ejected from galactic fountains return to the disk at average galactocentric distances several times larger than the galactocentric distance of the fountain. However, their mass resolution (particle mass $\simeq 1.5 \times 10^{5} M_{\odot}$ in the highest resolution simulation) makes their results more suitable for understanding 
Table 5. Radial flow velocities (in $\mathrm{km} \mathrm{s}^{-1}$ ) in the solar neighborhood (model without drag) as a function of the radial throwing coordinate.

\begin{tabular}{cccc}
\hline \hline & $4 \mathrm{kpc}$ & $8 \mathrm{kpc}$ & $12 \mathrm{kpc}$ \\
\hline $10 \mathrm{SNe}$ & 0.6 & 0.6 & 1.1 \\
$50 \mathrm{SNe}$ & 1.6 & 2.3 & 4.2 \\
$100 \mathrm{SNe}$ & 2.6 & 4.1 & 6.6 \\
$500 \mathrm{SNe}$ & 4.5 & 13.2 & 14.4 \\
\hline
\end{tabular}

the global behavior of the HI in the Galaxy rather than the evolution of a single superbubble/supershell. In Table 5 we report the main radial velocity for each fountains given by:

$\left\langle v_{R}\right\rangle=\frac{\langle\Delta R\rangle}{\langle\Delta t\rangle}$,

where $\Delta R=R_{\text {final }}-R_{0}$ and $\Delta t=t_{\text {final }}+t_{\text {orbit }}$; the time $t_{\text {orbit }}$ is the time required for the cloud to return to the galactic disk once it leaves the supershell. Several chemical evolution studies have suggested that the metallicity gradient disappears if the velocity of the radial flow is $\left|v_{\mathrm{f}}\right|>2 \mathrm{~km} \mathrm{~s}^{-1}$ (see Matteucci 2001).

For the model with viscous interaction we must know also the mass of the cloud because the drag terms depend on this quantity (see Eq. (37)). Referring to Fig. 1 we assume that the part of the shell that could fragment and move upwards is the mass included above the $s$ height where $\frac{\mathrm{d} R}{\mathrm{~d} z}=0$. Above this height each point of the shell has a positive velocity component along the $z$ axis. Therefore, we estimate that the masses of a gas cloud ejected by sequential SN events in the extra-planar halo are of the order of:

$M_{\text {cloud }_{R_{0}=4}}=2.17 \times 10^{5} M_{\odot}$,
$M_{\text {cloud }_{R_{0}=8}}=1.24 \times 10^{5} M_{\odot}$,
$M_{\text {cloud }_{R_{0}=12}}=0.84 \times 10^{5} M_{\odot}$.

We note that the masses of the clouds are roughly $20 \%$ of the initial supershell mass, therefore the total amount of the ejected metals in clouds is roughly $20 \%$ of the metals produced by the OB association, in agreement with the recent Melioli et al. (2008) hydrodynamical simulation results.

In Fig. 9 we show that for the range of initial velocities considered here, the effect of a viscous term in the motion equations is weak. The most evident effect is reported in Fig. 10 where the hybrid model is compared with the purely ballistic one in the case of 500 sequential SN explosions at $R_{0}=12 \mathrm{kpc}$. The natural effect of a viscous interaction between the cloud and the extraplanar gas is to brake the motion of the clouds. Therefore, the average radial coordinate falling on the disk for the model with drag $\left\langle R_{\text {final }}\right\rangle=12.37 \mathrm{kpc}$ is much smaller than the one obtained with the purely ballistic model $\left(\left\langle R_{\text {final }}\right\rangle=14.38 \mathrm{kpc}\right)$

As shown in Table 6 , it is likely that the most realistic number of massive stars in $\mathrm{OB}$ associations in our Galaxy is about 100. In Fig. 11 we have reproduced various fountains for the model without drag obtained with 100 sequential SN explosions respectively at $12,8,4 \mathrm{kpc}$ as radial coordinates. As shown before, different radial initial conditions imply different ejection velocities for the clouds. In particular, the throwing coordinate $R_{0}=12 \mathrm{kpc}$ is surrounded by a more tenuous disk, therefore the velocity is greater and the orbits wider. The highest $z$ coordinate that a cloud could reach in this case is about $z=2.3 \mathrm{kpc}$.

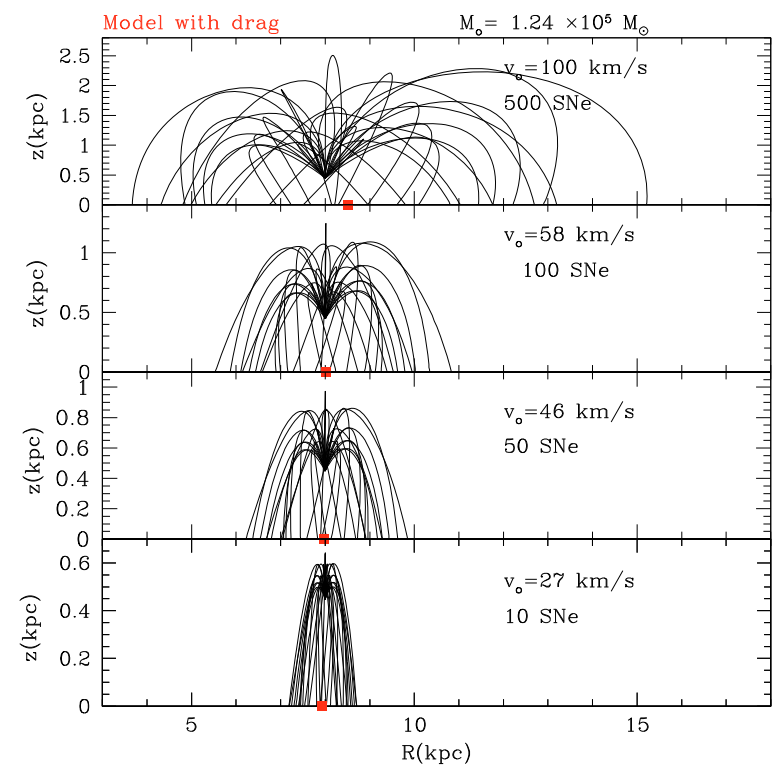

Fig. 9. Galactic fountains in a model with drag with the initial radial coordinate $R_{0}=8 \mathrm{kpc}$. In this case the orbits depend on the mass of the cloud ejected (indicated on the top of the panels). The natural effect of the viscous interaction is to decelerate the clouds.

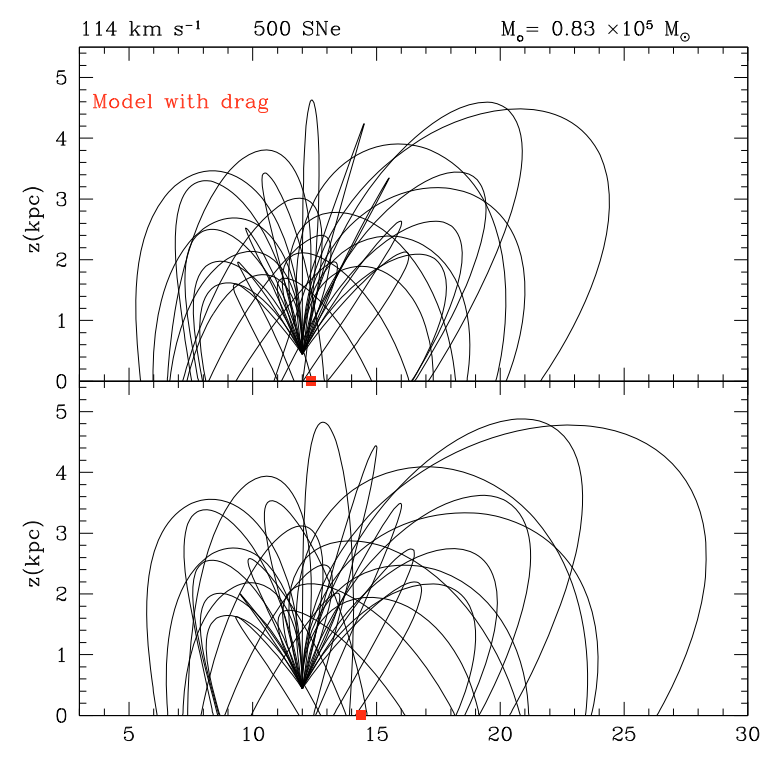

Fig. 10. Fountains that reach the maximum height in our model.

Table 6. Massive stars in OB associations from de Zeeuw et al. (1998).

\begin{tabular}{cccccc}
\hline \hline OB association & $W R$ & $O$ & $B$ & $A$ & Total \\
\hline Upper Scorpius & & & 49 & 34 & 83 \\
Upper Centaurus Lupus & & & 66 & 68 & 134 \\
Lower Centaurus Crux & & & 42 & 55 & 97 \\
Vela OB2 & 1 & & 81 & 5 & 87 \\
Trumpler 10 & & & 22 & 1 & 23 \\
Collinder 121 & 1 & 1 & 85 & 8 & 95 \\
Perseus OB2 & & & 17 & 16 & 33 \\
$\alpha$ Perseus OB2 & & & 33 & 30 & 63 \\
Lacerta OB1 & & 1 & 35 & 46 & 82 \\
Cepheus OB2 & & 1 & 56 & 10 & 67 \\
\hline
\end{tabular}




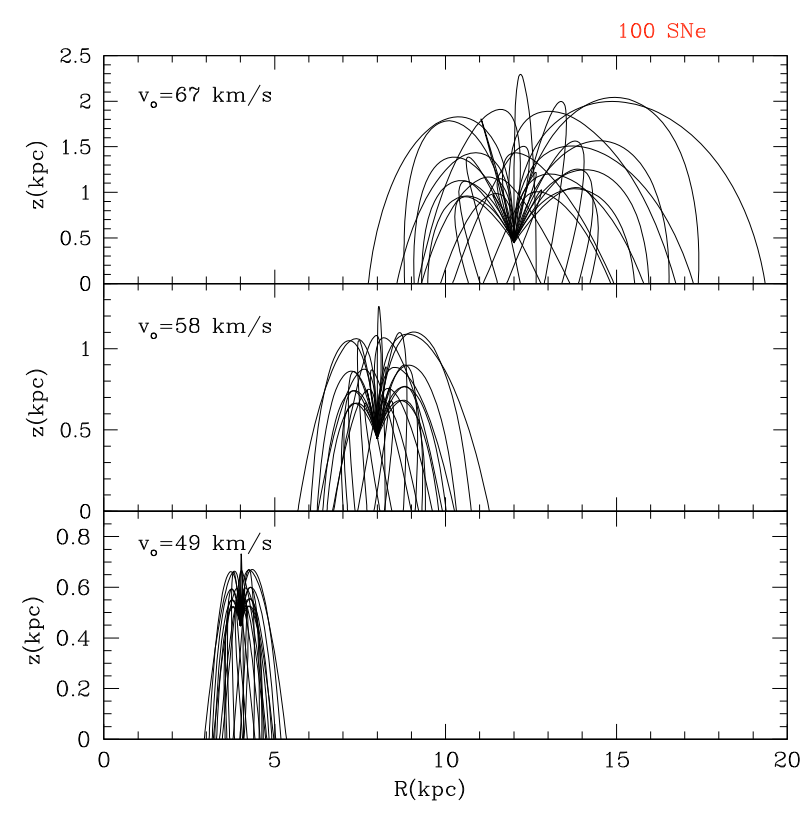

Fig. 11. Fountains in the purely ballistic model driven by a sequential explosion of $100 \mathrm{SNe}$.

\section{Comparison with HVC and IVC observations}

In this section we compare our models with observational data concerning distance and chemical composition of some clouds in our Galaxy. We consider two cloud systems: Complex C (HVCs) and IV Arch (IVC).

\subsection{Complex C}

In the work of Wakker et al. (2007) the first successful detection of interstellar $\mathrm{Ca}$ II $H$ and $\mathrm{K}$ absorption from HVC complex $\mathrm{C}$ was reported. They concluded that complex $\mathrm{C}$ is located at Galactocentric radius $<14 \mathrm{kpc}$, and lies high above the Galactic plane $(z=3-9 \mathrm{kpc})$.

Integrating the $H_{\mathrm{I}}$ column density across the cloud, $M(H \mathrm{I})$ was estimated as $0.7-6.0 \times 10^{6} M_{\odot}$. As we show in Eqs. (47)-(49), the sizes of our ejected clouds are considerably smaller. Even if we consider the case of the superbubble given by $500 \mathrm{SN}$ explosions with $R_{0}=12 \mathrm{kpc}$, the highest $z$ we can reach is about $z \simeq 4.4 \mathrm{kpc}$ and at this height our cloud velocities are roughly zero. In the Table 1 of Wakker et al. (2007) the velocities (relative to the LSR) of complex $\mathrm{C}$ are reported in the direction of different lines of sight, and on average the complex $\mathrm{C}$ velocity is $-114 \mathrm{~km} \mathrm{~s}^{-1}$. Therefore, the kinematical data of Complex $\mathrm{C}$ are inconsistent with our results.

A key point for understanding the origin of this $\mathrm{HVC}$ is to consider its chemical composition. Because oxygen is not significantly depleted onto dust grains (Meyer et al. 1998) and the $\mathrm{O} \mathrm{I} / \mathrm{H} \mathrm{I}$ ratio is not altered by ionization effects, we have that $(\mathrm{OI} / \mathrm{HI}) \approx(\mathrm{O} / \mathrm{H})$. In the work of Richter et al. (2001) the solar values for the abundances are given by Anders \& Grevesse (1989) and Grevesse \& Noels (1993). For oxygen they obtained:

$[\mathrm{O} / \mathrm{H}] \simeq[\mathrm{O} \mathrm{I} / \mathrm{HI}]=-1.03_{-0.31}^{+0.37}$.

For the iron abundance in the Complex C:

$[\mathrm{Fe} / \mathrm{H}] \simeq[(\mathrm{Fe} \mathrm{II}+\mathrm{Fe} \mathrm{III}) / \mathrm{HI}] .=-1.15$.
Based on the data from Fe II they obtained $[\mathrm{Fe} / \mathrm{H}]=-1.27_{-0.14}^{+0.20}$. Therefore a low iron abundance in Complex $\mathrm{C}$ could be a result of depletion of iron onto Fe rich dust (Savage \& Sembach 1996). A more careful discussion about the possible effects of dust is given in Sect. 4.3. Hence the relative abundance is $[\mathrm{O} / \mathrm{Fe}]=$ 0.12 . In our model a similar result is obtained in the case of $500 \mathrm{SNe}$ with the initial radial throwing coordinate fixed at $R_{0}=$ $12 \mathrm{kpc}$ and, as the disk initial gas metallicity, $Z=0.1 \times Z_{\odot}$. As we have seen, the most likely metallicity of the disk at $R_{0}=12 \mathrm{kpc}$ at the present time is $0.74 Z_{\odot}$, which is not consistent with the total metallicity of Complex C (Eqs. (50) and (51)). With our model we obtain $[\mathrm{O} / \mathrm{Fe}]=0.03 \mathrm{dex}$ in the case of $500 \mathrm{SNe}$ with a throwing coordinate $R_{0}=12 \mathrm{kpc}$ and metallicity fixed at $0.74 Z_{\odot}$ (see Fig. 7). Such an oxygen overabundance, although not impossible, is quite unlikely. Given the inconsistency of the kinematical data with our predictions, we can rule out a Galactic origin for the Complex C HVC.

\subsection{ARCH}

Richter et al. (2001) suggested that IV Arch, given its nearly solar abundance and its $z$-height of $0.8-1.5 \mathrm{kpc}$, has its origin in the Milky Way disk, probably as part of a Galactic fountain. These heights are consistent with our results, and also the velocities of IVCs are easily understandable in the framework of galactic fountains. The abundances measured by Richter et al. (2001) are: $[\mathrm{O} / \mathrm{H}]=-0.01_{-0.27}^{+0.35}$ and $[\mathrm{Fe} / \mathrm{H}]=-0.26_{-0.15}^{+0.19}$, therefore:

$[\mathrm{O} / \mathrm{Fe}]=[\mathrm{O} / \mathrm{H}]-[\mathrm{Fe} / \mathrm{H}]=0.25 \mathrm{dex}$.

If we assume a Galactic fountain origin, our model yields $[\mathrm{O} / \mathrm{Fe}]=0.24 \mathrm{dex}$ in the case of $500 \mathrm{SN}$ explosions, $R_{0}=8 \mathrm{kpc}$ and metallicity $Z=0.01 \times Z_{\odot}$, and $[\mathrm{O} / \mathrm{Fe}]=0.26 \mathrm{dex}$ in the case of $100 \mathrm{SN}$ explosions, $R_{0}=12 \mathrm{kpc}$ and metallicity $Z=0.01 \times Z_{\odot}$. As we have seen, it is quite unlikely that the initial metallicity of an OB association is nowadays as low as $0.01 Z_{\odot}$ and this low initial metallicity would be inconsistent with the nearly solar abundance measured in IV Arch. Therefore, although the kinematic properties of IV Arch are in agreement with our results, its large $[\mathrm{O} / \mathrm{Fe}]$ casts some doubt about the Galactic origin of this IVC.

\subsection{The effect of dust}

The work of Lu et al. (1998) showed how important the depletion of metals (in particular Fe) in the ISM is into dust. Since this can affect out results about $[\mathrm{O} / \mathrm{Fe}]$ ratios, here we estimate the time scales of the destruction and accretion of dust in a superbubble. Referring to the work of Calura et al. (2008) we define, for a given element $i$ the destruction time-scale $\tau_{\text {destr }}$ for the dust in the superbubble due to $\mathrm{SN}$ shocks as:

$\tau_{\mathrm{destr}, i}=\left(\epsilon M_{\mathrm{SNR}}\right)^{-1} \cdot \frac{\sigma_{\mathrm{ISM}}}{R_{\mathrm{SN}}}$,

where $R_{\mathrm{SN}}$ is the total SN rate, $M_{\mathrm{SNR}}$ is the mass of the interstellar gas swept up by the SN remnant and $\epsilon$ is the destruction efficiency in a three-phase medium. We consider for $\sigma_{\text {ISM }}$, the surface gas density of the ISM, the value of $10 M_{\odot} \mathrm{pc}^{-2}$ and $\epsilon M_{\mathrm{SNR}} \simeq 10^{5} M_{\odot}$. For the SN rate we consider the typical case of the OB association with $100 \mathrm{SNe}$. We assume as the radius of the OB association the conservative value of $100 \mathrm{pc}$. We obtain that $\tau_{\text {destr, } i} \simeq 0.7 \mathrm{Myr}$. This timescale is considerably shorter than the time necessary for the formation of a RT instable supershell in our model (See Sect. 3.1). If the number of SN is smaller, $\tau_{\text {destr }}$ is larger but it remains considerably smaller than 
$t_{\text {final }}$. Given the complexity of the interaction between SN shocks and dust (Draine \& Salpeter 1979; Jones et al. 1996) this estimate is necessarily simplified but it leads to the suggestion that only a small amount of dust can survive in the supershell swept up by the OB association, therefore only a small fraction of metals in the clouds driven by the galactic fountains is locked into dust grains. Can this amount significantly increase by dust accretion during the journey of the cloud? To answer this question we have to compare the dynamical timescale of our clouds with the typical dust accretion timescale.

Following the work of Calura et al. (2008), let $X_{\mathrm{d}}$ be the abundance by mass of the dust and $\sigma$ the ISM fraction at time $t$, the quantity $\sigma_{\text {dust }}=X_{\mathrm{d}} \cdot \sigma$ represents the normalized mass density of the dust at the time $t$.

The accretion timescale $\tau_{\text {accr }}$ is given by:

$\tau_{\text {accr }}=\tau_{0} /\left(1-X_{\mathrm{d}}\right)$,

for $\tau_{0}$ typical values range between $50 \mathrm{Myr}$ and $200 \mathrm{Myr}$ (see Dwek 1998). The integration of the equation for the temporal evolution of $X_{\mathrm{d}}$ with the initial condition $X_{\mathrm{d}, 0} \simeq 0$ and $X_{\mathrm{d}} \ll 1$ gives:

$t \simeq \tau_{0, i}\left[\ln \left(X_{\mathrm{d}} / X_{\mathrm{d}, 0}\right)-\ln (1)\right]$.

We want to know how much time it takes to increase the initially small amount of dust up to a non-negligible value. If we calculate for instance the time necessary to accrete 10 times the initial dust fraction (e.g. $\left.X_{\mathrm{d}} / X_{\mathrm{d}, 0}=10\right)$, we get:

$t \simeq 2.3 \tau_{0, i}$.

This time is greater than the average time of the cloud orbits, therefore we can conclude that the depletion of metals into dust does not play a important role in the supershell evolution.

\section{Conclusions}

In this paper we have studied the evolution of a supershell powered by the energetic feedback of a typical Galactic OB association at various Galactocentric radii. Based on the Kompaneets (1960) approximation, we have found analytical solutions for the temporal evolution of the supershell and we have established criteria for its fragmentation, which can create clouds that are thrown out of the disk. Given the self-similar behavior of the Kompaneets solutions, the clouds are formed at the same scale height $(\sim 450 \mathrm{pc})$, irrespective of the number of $\mathrm{SNe}$ in the OB association or of the Galactocentric radius at which the $\mathrm{OB}$ association lies. Assuming that the ejecta of the dying stars of the OB association instantaneously mix with the supershell, we are able to calculate the chemical composition of the clouds and in particular their $[\mathrm{O} / \mathrm{Fe}]$.

We have considered four different $\mathrm{OB}$ associations (containing 10, 50, 100 and $500 \mathrm{SNe}$, respectively) and three different initial throwing coordinates (4, 8 and $12 \mathrm{kpc}$, respectively). Once the clouds are formed and can leave the disk, we follow their orbits either assuming a purely ballistic model, or introducing a viscous force acting between the cloud and the surrounding hot halo gas. Our main conclusions can be summarized as follows:

- If the initial metallicity of the OB association is solar, the pollution from the dying stars has a negligible effect on the chemical composition of the clouds. In particular, the $[\mathrm{O} / \mathrm{Fe}]$ abundance ratio reaches at most $\sim 0.025$ in a model in which the throwing coordinate is $R_{0}=12 \mathrm{kpc}$. Only starting from very low metallicities (less than $1 / 100 Z_{\odot}$ ) it is possible to produce a significant enrichment of $\alpha$-elements.
- Both in the ballistic and in the viscous interaction models the maximum height reached by the clouds is not very large. Only for OB associations composed of $500 \mathrm{SNe}$ is it possible to eject clouds up to heights larger than $2 \mathrm{kpc}$ above the plane of the Galaxy.

- The range of the cloud orbits is also quite small. The clouds are generally directed outwards but the average landing coordinates differ from the throwing coordinates by $\sim 1 \mathrm{kpc}$ at most. Only for a throwing coordinate of $12 \mathrm{kpc}$ and an OB association made of $500 \mathrm{SNe}$ does the ballistic model predict a landing coordinate $\sim 2 \mathrm{kpc}$ larger than the throwing one.

- Models including a viscous interaction between clouds and the extra-planar gas predict smaller ranges of the cloud orbits. Indeed, the drag experienced by the cloud brakes it and therefore it shortens its journey above the Galactic plane.

- The HVC Complex C has a mass, velocity and inferred height above the plane inconsistent with the results of our models. Its oxygen overabundance $([\mathrm{O} / \mathrm{Fe}]=0.12)$ can be reproduced only if we assume a large $\mathrm{OB}$ association with metallicity $0.1 Z_{\odot}$ or smaller, therefore its Galactic origin cannot be completely ruled out on the basis of its chemical composition alone, but the kinematical data suggest a different formation mechanism. The IVC Arch IV instead has velocities and heights above the plane that are easily reproduced by our models, but its $[\mathrm{O} / \mathrm{Fe}]=0.25$ is much larger than the one of Complex $\mathrm{C}$ and it can be explained only by assuming initial metallicities smaller than $1 / 100 Z_{\odot}$, which are unlikely at the present time for the Galactic disk. Therefore, it is unlikely that the two studied clouds originated in a Galactic fountain motion.

Acknowledgements. We thank the referee for the enlightening suggestions. We are grateful to A. D'Ercole for having kindly provided a ballistic galactic fountains code. We also thank F. Calura, G. Cescutti and A. Palestrini for many useful discussions.

\section{References}

Anders, E., \& Grevesse, N. 1989, Geochim. Cosmochim. Acta, 53, 197 Andrievsky, S. M., Bersier, D., Kovtyukh, V. V., et al 2002a, A\&A, 384, 140 Andrievsky, S. M., Kovtyukh, V. V., Luck, R. E., et al. 2002b, A\&A, 381, 32 Andrievsky, S. M., Kovtyukh, V. V., Luck, R. E., et al. 2002c, A\&A, 392, 491 Andrievsky, S. M., Luck, R. E., Martin, P., et al. 2004, A\&A, 413, 159

Asplund, M., Grevesse, N., \& Sauval, A. J. 2005, in Cosmic Abundances as Records of Stellar Evolution and Nucleosynthesis, ed. T. G. Barnes III, \& F. N., Bash, ASP Conf. Ser., 336, 25

Booth, C. M., \& Theuns, T. 2007, MNRAS, 381, 89

Barnabè, M., Ciotti, L., Fraternali, F., \& Sancisi, R. 2006, A\&A, 446, 61 Basu, S., Johnstone, D., \& Martin, P. G. 1999, ApJ, 516, 843

Blitz, L., Spergel, D. N., Teuben, P. J., Hartmann, D., \& Burton, W. B. 1999, ApJ, 514, 818

Bluhm, H., de Boer, K., Marggraf, O., \& Richter, P. 2001, A\&A, 367, 299

Boomsma, R., Oosterloo, T., Fraternali, F., van der Hulst, T., \& Sancisi, R. 2005, in Extra-planar Gas Conference, ASP Conf. Ser., ed. R. Braun, 331, 247 Bregman, J. N. 1980, ApJ, 365, 544

Calura, F., Pipino, A., \& Matteucci, F. 2008, A\&A, 479, 669

Cescutti, G., Matteucci, F., Francois, P., \& Chiappini, C. 2006, A\&A, 462, 943 Charlton, J. C., \& Salpeter, E. E. 1989, ApJ, 346,101

Chiappini, C., Matteucci, F., \& Romano, D. 2001, ApJ, 554, 1044 Collins, J. A., Benjamin, R. A., \& Rand, R. J. 2002, ApJ, 578, 98 Cox, D. P. 2005, ARA\&A, 43, 337

de Zeeuw, P. T., Hoogerwerf, R., \& de Bruijne, J. H. J. 1999, ApJ, 117, 354 Draine, B. T., \& Salpeter, E. E. 1979, ApJ, 231, 438

Dwek, E. 1998, ApJ, 501, 643

Fraternali, F., \& Oosterloo, T., \& Sancisi, R. 2004, A\&A, 424, 485

Fraternali, F., \& Binney, J. 2008, MNRAS, accepted [arXiv: 0802 . 0496]

Grevesse, N., \& Noels, A. 1993, in Orgin of the Elements, ed. N. Prantzos, E. Vangioni-Flam, \& M. Cassé (Cambridge: Univ. Press), 15 
Hernquist, L. 1990, ApJ, 356, 359

Houck, J. C., \& Bregman, J. N. 1990, ApJ, 352, 506

Ibata, R. A., Gilmore, G., \& Irwin, M. J. 1994, Nature, 370, 194

Kompaneets, A. S. 1960, Soviet Phys. Dokl., 5, 46

Jones, A. P., Tielens, G. G. M., \& Hollenbach, D. J. 1996, ApJ, 469, 740

Lu, L., Sargent, W. L. W., Savage, B. D., et al. 1998, AJ, 115, 162

Luck, R. E., Gieren, W. P., Andrievsky, S. M., et al. 2003, A\&A, 401, 939

Mac Low, M. M., \& McCray, R. 1988. ApJ, 324, 776

Matteucci, F. 2001, The Chemical Evolution Of The Galaxy (Kluwer Academic Publishers)

Matteucci, F., \& Greggio, L. 1986, A\&A, 154, 279

Melioli, C., Brighenti, F., D’Ercole, A., \& de Gouveia Dal Pino, E. M. 2008, MNRAS, accepted [arXiv:0805.1138]

Miyamoto, M., \& Nagai, R. 1975, PASJ, 27, 533
Murphy, E. M., Sembach, K. R., Gibson, B. K., et al. 2000, ApJ, 538, L35 Navarro, J. D., Frenk, C. S., \& White, S. D. M. 1996, ApJ, 462, 563

Oort, J. H. 1970, A\&A, 7, 381

Richter, P., Sembach, K. R., Wakker, B. P., et al. 2001, ApJ, 559, 318

Salpeter, E. E. 1955, ApJ, 121, 161

Sembach, K. R., \& Oosterloo, T. A. 1998, AJ, 115, 162

Sembach, K. R., Howk, J. C., Savage, B. D., \& Shull, J. M. 2001, AJ, 121, 992 Shapiro, P. R., \& Field, G. B. 1976, ApJ 205, 762

Tenorio-Tagle, G., Bodenheimer, P., \& Różyczka, M. 1987, A\&A, 182, 120

Tenorio-Tagle, G., Silich, S. A., Kunth, D., Terlevich, E., \& Terlevich, R. 1999, MNRAS, 309, 332

Wakker, B. P., York, D. G., Howk, C., et al. 2007, ApJ, 670, 113

Wakker, B. P., Howk, J. C., Savage, B. D., et al. 1999, Nature, 402, 388

Woosley, S. E., \& Weaver, T. A. 1995, ApJ, 101, 181 
E. Spitoni et al.: Galactic fountains, Online Material $p 1$

\section{Appendix A}

In the following tables we summarize all our results concerning the abundances of oxygen and iron calculated with our model for the initial throwing radial coordinates $R_{0}=4,8,12 \mathrm{kpc}$ and for metallicities: $Z_{\odot}, 0.1 \times Z_{\odot}, 0.01 \times Z_{\odot}, 10^{-4} \times Z_{\odot}$. 
Table A.1. $Z=Z_{\odot} 8 \mathrm{kpc}$.

\begin{tabular}{cccccccccc}
\hline \hline $\begin{array}{c}\text { Number } \\
\text { of SNe }\end{array}$ & $M_{* \mathrm{Fe}_{56}}$ & $M_{* \mathrm{O}_{16}}$ & $M_{* \mathrm{ej}}$ & $M_{\text {ShellFe }_{56}}$ & $M_{\text {ShellO }_{16}}$ & $M_{\text {tot }}$ & $X_{* \mathrm{Fe}_{56}}$ & $X_{* \mathrm{O}_{16}}$ & {$[\mathrm{O} / \mathrm{Fe}]$} \\
\hline 10 & 0.95 & 10.28 & $1.16 \times 10^{2}$ & 678.40 & $5.56 \times 10^{3}$ & $57.91 \times 10^{4}$ & $1.17 \times 10^{-3}$ & $9.60 \times 10^{-3}$ & $1.97 \times 10^{-4}$ \\
50 & 3.95 & 49.89 & $4.77 \times 10^{2}$ & 681.40 & $5.60 \times 10^{3}$ & $57.94 \times 10^{4}$ & $1.17 \times 10^{-3}$ & $9.67 \times 10^{-3}$ & $1.36 \times 10^{-3}$ \\
100 & 6.99 & 97.55 & $8.52 \times 10^{2}$ & 684.44 & $5.69 \times 10^{3}$ & $57.98 \times 10^{4}$ & $1.18 \times 10^{-3}$ & $9.75 \times 10^{-3}$ & $3.11 \times 10^{-3}$ \\
500 & 24.69 & 437.46 & $3.18 \times 10^{3}$ & 702.14 & $5.99 \times 10^{3}$ & $58.22 \times 10^{4}$ & $1.21 \times 10^{-3}$ & $1.03 \times 10^{-2}$ & $1.74 \times 10^{-2}$ \\
\hline
\end{tabular}

Table A.2. $Z=0.1 \times Z_{\odot} 8 \mathrm{kpc}$.

\begin{tabular}{cccccccccc}
\hline \hline $\begin{array}{c}\text { Number } \\
\text { of SNe }\end{array}$ & $M_{* \mathrm{Fe}_{56}}$ & $M_{* \mathrm{O}_{16}}$ & $M_{* \mathrm{ej}}$ & $M_{\mathrm{ShellFe}_{56}}$ & $M_{\text {ShellO }_{16}}$ & $M_{\mathrm{tot}}$ & $X_{* \mathrm{Fe}_{56}}$ & $X_{* \mathrm{O}_{16}}$ & {$[\mathrm{O} / \mathrm{Fe}]$} \\
\hline 10 & 0.95 & 9.33 & $1.14 \times 10^{2}$ & 68.70 & $5.64 \times 10^{2}$ & $57.91 \times 10^{4}$ & $1.19 \times 10^{-4}$ & $9.75 \times 10^{-4}$ & $1.17 \times 10^{-3}$ \\
50 & 3.95 & 45.48 & $4.81 \times 10^{2}$ & 71.70 & $6.01 \times 10^{2}$ & $57.95 \times 10^{4}$ & $1.24 \times 10^{-4}$ & $1.04 \times 10^{-3}$ & $9.55 \times 10^{-3}$ \\
100 & 6.99 & 88.97 & $8.59 \times 10^{2}$ & 75.57 & $6.44 \times 10^{2}$ & $57.99 \times 10^{4}$ & $1.30 \times 10^{-4}$ & $1.11 \times 10^{-3}$ & $1.71 \times 10^{-2}$ \\
500 & 24.69 & 401.60 & $3.21 \times 10^{3}$ & 92.43 & $9.57 \times 10^{2}$ & $58.22 \times 10^{4}$ & $1.59 \times 10^{-4}$ & $1.64 \times 10^{-3}$ & $1.01 \times 10^{-1}$ \\
\hline
\end{tabular}

Table A.3. $Z=0.01 \times Z_{\odot} 8 \mathrm{kpc}$.

\begin{tabular}{cccccccccc}
\hline \hline $\begin{array}{c}\text { Number } \\
\text { of SNe }\end{array}$ & $M_{* \mathrm{Fe}_{56}}$ & $M_{* \mathrm{O}_{16}}$ & $M_{* \mathrm{ej}}$ & $M_{\text {ShellFe }_{56}}$ & $M_{\text {ShellO }_{16}}$ & $M_{\text {tot }}$ & $X_{* \mathrm{Fe}_{56}}$ & $X_{* \mathrm{O}_{16}}$ & {$[\mathrm{O} / \mathrm{Fe}]$} \\
\hline 10 & 0.95 & 8.96 & $1.14 \times 10^{2}$ & 7.72 & 64.50 & $57.91 \times 10^{4}$ & $1.33 \times 10^{-5}$ & $1.11 \times 10^{-4}$ & $8.29 \times 10^{-3}$ \\
50 & 3.95 & 43.67 & $4.82 \times 10^{2}$ & 10.73 & 99.20 & $57.95 \times 10^{4}$ & $1.85 \times 10^{-5}$ & $1.71 \times 10^{-4}$ & $5.23 \times 10^{-2}$ \\
100 & 6.99 & 85.72 & $8.61 \times 10^{2}$ & 13.77 & $1.41 \times 10^{2}$ & $57.99 \times 10^{4}$ & $2.37 \times 10^{-5}$ & $2.43 \times 10^{-4}$ & $9.74 \times 10^{-2}$ \\
500 & 24.69 & 394.21 & $3.23 \times 10^{3}$ & 31.46 & $4.50 \times 10^{2}$ & $58.22 \times 10^{4}$ & $5.40 \times 10^{-5}$ & $7.72 \times 10^{-4}$ & $2.41 \times 10^{-1}$ \\
\hline
\end{tabular}

Table A.4. $Z=10^{-4} \times Z_{\odot} 8 \mathrm{kpc}$.

\begin{tabular}{cccccccccc}
\hline \hline $\begin{array}{c}\text { Number } \\
\text { of SNe }\end{array}$ & $M_{* \mathrm{Fe}_{56}}$ & $M_{* \mathrm{O}_{16}}$ & $M_{* \mathrm{ej}}$ & $M_{\text {ShellFe }_{56}}$ & $M_{\text {ShellO }_{16}}$ & $M_{\text {tot }}$ & $X_{* \mathrm{Fe}_{56}}$ & $X_{* \mathrm{O}_{16}}$ & {$[\mathrm{O} / \mathrm{Fe}]$} \\
\hline 10 & 0.95 & 8.55 & $1.14 \times 10^{2}$ & 1.02 & 9.10 & $57.91 \times 10^{4}$ & $2.09 \times 10^{-6}$ & $1.57 \times 10^{-5}$ & $3.68 \times 10^{-2}$ \\
50 & 3.95 & 41.54 & $4.81 \times 10^{2}$ & 4.02 & 42.10 & $57.95 \times 10^{4}$ & $7.26 \times 10^{-6}$ & $7.26 \times 10^{-5}$ & $1.06 \times 10^{-1}$ \\
100 & 6.99 & 81.58 & $8.60 \times 10^{2}$ & 7.06 & 82.14 & $57.99 \times 10^{4}$ & $1.22 \times 10^{-5}$ & $1.42 \times 10^{-4}$ & $1.52 \times 10^{-1}$ \\
500 & 24.69 & 377.66 & $3.23 \times 10^{3}$ & 24.75 & $3.78 \times 10^{2}$ & $58.22 \times 10^{4}$ & $4.25 \times 10^{-5}$ & $6.52 \times 10^{-4}$ & $2.70 \times 10^{-1}$ \\
\hline
\end{tabular}

Table A.5. $Z=Z_{\odot} 12 \mathrm{kpc}$.

\begin{tabular}{cccccccccc}
\hline \hline $\begin{array}{c}\text { Number } \\
\text { of SNe }\end{array}$ & $M_{* \mathrm{Fe}_{56}}$ & $M_{* \mathrm{O}_{16}}$ & $M_{* \mathrm{ej}}$ & $M_{\text {ShellFe } 56_{6}}$ & $M_{\text {Shell }_{16}}$ & $M_{\text {tot }}$ & $X_{* \mathrm{Fe}_{56}}$ & $X_{* \mathrm{O}_{16}}$ & {$[\mathrm{O} / \mathrm{Fe}]$} \\
\hline 10 & 0.92 & 10.23 & $1.13 \times 10^{2}$ & 456.70 & $3.75 \times 10^{3}$ & $38.97 \times 10^{4}$ & $1.17 \times 10^{-3}$ & $9.61 \times 10^{-3}$ & $3.13 \times 10^{-4}$ \\
50 & 3.74 & 49.28 & $4.47 \times 10^{2}$ & 459.52 & $3.78 \times 10^{3}$ & $39.00 \times 10^{4}$ & $1.18 \times 10^{-3}$ & $9.70 \times 10^{-3}$ & $2.14 \times 10^{-3}$ \\
100 & 6.35 & 95.73 & $7.95 \times 10^{2}$ & 462.13 & $3.83 \times 10^{3}$ & $39.03 \times 10^{4}$ & $1.18 \times 10^{-3}$ & $9.81 \times 10^{-3}$ & $4.98 \times 10^{-3}$ \\
500 & 23.27 & 421.95 & $2.95 \times 10^{3}$ & 479.05 & $4.16 \times 10^{3}$ & $39.25 \times 10^{4}$ & $1.22 \times 10^{-3}$ & $1.06 \times 10^{-2}$ & $2.48 \times 10^{-2}$ \\
\hline
\end{tabular}


Table A.6. $Z=0.1 \times Z_{\odot} 12 \mathrm{kpc}$.

\begin{tabular}{cccccccccc}
\hline \hline $\begin{array}{c}\text { Number } \\
\text { of SNe }\end{array}$ & $M_{* \mathrm{Fe}_{56}}$ & $M_{* \mathrm{O}_{16}}$ & $M_{* \mathrm{ej}}$ & $M_{\text {ShellFe }_{56}}$ & $M_{\text {ShellO }_{16}}$ & $M_{\mathrm{tot}}$ & $X_{* \mathrm{Fe}_{56}}$ & $X_{* \mathrm{O}_{16}}$ & {$[\mathrm{O} / \mathrm{Fe}]$} \\
\hline 10 & 0.92 & 9.29 & $1.11 \times 10^{2}$ & 46.50 & $3.83 \times 10^{2}$ & $38.97 \times 10^{4}$ & $1.19 \times 10^{-4}$ & $9.82 \times 10^{-4}$ & $1.98 \times 10^{-3}$ \\
50 & 3.74 & 45.00 & $4.51 \times 10^{2}$ & 49.32 & $4.18 \times 10^{2}$ & $39.00 \times 10^{4}$ & $1.26 \times 10^{-4}$ & $1.07 \times 10^{-3}$ & $1.51 \times 10^{-2}$ \\
100 & 6.35 & 87.27 & $8.02 \times 10^{2}$ & 51.92 & $4.61 \times 10^{2}$ & $39.03 \times 10^{4}$ & $1.33 \times 10^{-4}$ & $1.18 \times 10^{-3}$ & $3.46 \times 10^{-2}$ \\
500 & 23.27 & 388.11 & $2.97 \times 10^{3}$ & 68.85 & $7.62 \times 10^{2}$ & $39.25 \times 10^{4}$ & $1.75 \times 10^{-4}$ & $1.94 \times 10^{-3}$ & $1.30 \times 10^{-1}$ \\
\hline
\end{tabular}

Table A.7. $Z=0.01 \times Z_{\odot} 12 \mathrm{kpc}$.

\begin{tabular}{cccccccccc}
\hline \hline $\begin{array}{c}\text { Number } \\
\text { of SNe }\end{array}$ & $M_{* \mathrm{Fe}_{56}}$ & $M_{* \mathrm{O}_{16}}$ & $M_{* \mathrm{ej}}$ & $M_{\text {ShellFe }_{56}}$ & $M_{\text {ShellO }_{16}}$ & $M_{\mathrm{tot}}$ & $X_{* \mathrm{Fe}_{56}}$ & $X_{* \mathrm{O}_{16}}$ & {$[\mathrm{O} / \mathrm{Fe}]$} \\
\hline 10 & 0.92 & 8.93 & $1.11 \times 10^{2}$ & 5.48 & 46.29 & $38.97 \times 10^{4}$ & $1.41 \times 10^{-5}$ & $1.19 \times 10^{-4}$ & $1.31 \times 10^{-2}$ \\
50 & 3.74 & 43.24 & $4.52 \times 10^{2}$ & 8.30 & 80.60 & $39.00 \times 10^{4}$ & $2.13 \times 10^{-5}$ & $2.07 \times 10^{-4}$ & $7.36 \times 10^{-2}$ \\
100 & 6.35 & 84.64 & $8.06 \times 10^{2}$ & 10.90 & 121.82 & $39.04 \times 10^{4}$ & $2.79 \times 10^{-5}$ & $3.12 \times 10^{-4}$ & $1.35 \times 10^{-1}$ \\
500 & 23.27 & 381.99 & $3.00 \times 10^{3}$ & 27.83 & 419.35 & $39.25 \times 10^{4}$ & $7.09 \times 10^{-5}$ & $1.07 \times 10^{-3}$ & $2.64 \times 10^{-1}$ \\
\hline
\end{tabular}

Table A.8. $Z=10^{-4} \times Z_{\odot} 12 \mathrm{kpc}$.

\begin{tabular}{cccccccccc}
\hline \hline $\begin{array}{c}\text { Number } \\
\text { of SNe }\end{array}$ & $M_{* \mathrm{Fe}_{56}}$ & $M_{* \mathrm{O}_{16}}$ & $M_{* \mathrm{ej}}$ & $M_{\text {ShellFe }_{66}}$ & $M_{\text {ShellO }_{16}}$ & $M_{\text {tot }}$ & $X_{* \mathrm{Fe}_{56}}$ & $X_{* \mathrm{O}_{16}}$ & {$[\mathrm{O} / \mathrm{Fe}]$} \\
\hline 10 & 0.92 & 8.51 & $1.11 \times 10^{2}$ & 0.97 & 8.88 & $38.97 \times 10^{4}$ & $2.49 \times 10^{-6}$ & $2.28 \times 10^{-5}$ & $4.80 \times 10^{-2}$ \\
50 & 3.74 & 41.13 & $4.52 \times 10^{2}$ & 3.79 & 41.5 & $39.00 \times 10^{4}$ & $9.72 \times 10^{-6}$ & $1.06 \times 10^{-4}$ & $1.26 \times 10^{-1}$ \\
100 & 6.35 & 80.47 & $8.05 \times 10^{2}$ & 6.39 & 80.84 & $39.04 \times 10^{4}$ & $1.64 \times 10^{-5}$ & $2.07 \times 10^{-4}$ & $1.88 \times 10^{-1}$ \\
500 & 23.27 & 367.43 & $2.99 \times 10^{3}$ & 23.31 & 367.81 & $39.25 \times 10^{4}$ & $5.94 \times 10^{-5}$ & $9.37 \times 10^{-4}$ & $2.84 \times 10^{-1}$ \\
\hline
\end{tabular}

Table A.9. $Z=Z_{\odot} 4$ kpc.

\begin{tabular}{cccccccccc}
\hline \hline $\begin{array}{c}\text { Number } \\
\text { of SNe }\end{array}$ & $M_{* \mathrm{Fe}_{56}}$ & $M_{* \mathrm{O}_{16}}$ & $M_{* \mathrm{ej}}$ & $M_{\text {ShellFe }_{56}}$ & $M_{\mathrm{ShellO}_{16}}$ & $M_{\text {tot }}$ & $X_{* \mathrm{Fe}_{56}}$ & $X_{* \mathrm{O}_{16}}$ & {$[\mathrm{O} / \mathrm{Fe}]$} \\
\hline 10 & 0.97 & 10.32 & $1.19 \times 10^{2}$ & 1179.14 & $9.67 \times 10^{3}$ & $100.71 \times 10^{4}$ & $1.17 \times 10^{-3}$ & $9.60 \times 10^{-3}$ & $1.04 \times 10^{-4}$ \\
50 & 4.26 & 50.57 & $5.20 \times 10^{2}$ & 1182.42 & $9.71 \times 10^{3}$ & $100.75 \times 10^{4}$ & $1.17 \times 10^{-3}$ & $9.63 \times 10^{-3}$ & $7.02 \times 10^{-4}$ \\
100 & 7.79 & 99.37 & $9.33 \times 10^{2}$ & 1185.95 & $9.76 \times 10^{3}$ & $100.79 \times 10^{4}$ & $1.18 \times 10^{-3}$ & $9.69 \times 10^{-3}$ & $1.58 \times 10^{-3}$ \\
500 & 27.36 & 457.84 & $3.53 \times 10^{3}$ & 1205.52 & $10.11 \times 10^{3}$ & $101.05 \times 10^{4}$ & $1.19 \times 10^{-3}$ & $1.00 \times 10^{-2}$ & $1.01 \times 10^{-2}$ \\
\hline
\end{tabular}

Table A.10. $Z=0.1 \times Z_{\odot} 4 \mathrm{kpc}$.

\begin{tabular}{cccccccccc}
\hline \hline $\begin{array}{c}\text { Number } \\
\text { of SNe }\end{array}$ & $M_{* \mathrm{Fe}_{56}}$ & $M_{* \mathrm{O}_{16}}$ & $M_{* \mathrm{ej}}$ & $M_{\text {ShellFe }_{56}}$ & $M_{\mathrm{ShellO}_{16}}$ & $M_{\mathrm{tot}}$ & $X_{* \mathrm{Fe}_{56}}$ & $X_{* \mathrm{O}_{16}}$ & {$[\mathrm{O} / \mathrm{Fe}]$} \\
\hline 10 & 0.97 & 9.36 & $1.16 \times 10^{2}$ & 118.79 & $9.75 \times 10^{2}$ & $100.71 \times 10^{4}$ & $1.18 \times 10^{-4}$ & $9.68 \times 10^{-4}$ & $6.17 \times 10^{-4}$ \\
50 & 4.26 & 45.99 & $5.19 \times 10^{2}$ & 122.08 & $10.11 \times 10^{2}$ & $100.75 \times 10^{4}$ & $1.21 \times 10^{-4}$ & $1.00 \times 10^{-3}$ & $4.77 \times 10^{-3}$ \\
100 & 7.79 & 90.67 & $9.41 \times 10^{2}$ & 125.61 & $10.56 \times 10^{2}$ & $100.79 \times 10^{4}$ & $1.25 \times 10^{-4}$ & $1.05 \times 10^{-3}$ & $1.11 \times 10^{-2}$ \\
500 & 27.36 & 418.89 & $3.56 \times 10^{3}$ & 145.17 & $13.84 \times 10^{2}$ & $101.05 \times 10^{4}$ & $1.44 \times 10^{-4}$ & $1.37 \times 10^{-3}$ & $6.58 \times 10^{-2}$ \\
\hline
\end{tabular}


Table A.11. $Z=0.01 \times Z_{\odot} 4 \mathrm{kpc}$.

\begin{tabular}{cccccccccc}
\hline \hline $\begin{array}{c}\text { Number } \\
\text { of SNe }\end{array}$ & $M_{* \mathrm{Fe}_{56}}$ & $M_{* \mathrm{O}_{16}}$ & $M_{* \mathrm{ej}}$ & $M_{\text {ShellFe }_{56}}$ & $M_{\text {ShellO }_{16}}$ & $M_{\mathrm{tot}}$ & $X_{* \mathrm{Fe}_{56}}$ & $X_{* \mathrm{O}_{16}}$ & {$[\mathrm{O} / \mathrm{Fe}]$} \\
\hline 10 & 0.97 & 9.00 & $1.16 \times 10^{2}$ & 12.76 & $1.05 \times 10^{2}$ & $100.71 \times 10^{4}$ & $1.27 \times 10^{-5}$ & $1.05 \times 10^{-4}$ & $4.06 \times 10^{-3}$ \\
50 & 4.26 & 44.18 & $5.20 \times 10^{2}$ & 16.04 & $1.41 \times 10^{2}$ & $100.75 \times 10^{4}$ & $1.59 \times 10^{-5}$ & $1.40 \times 10^{-4}$ & $2.96 \times 10^{-2}$ \\
100 & 7.79 & 87.06 & $9.43 \times 10^{2}$ & 19.57 & $1.84 \times 10^{2}$ & $100.79 \times 10^{4}$ & $1.94 \times 10^{-5}$ & $1.82 \times 10^{-4}$ & $5.87 \times 10^{-2}$ \\
500 & 27.36 & 408.93 & $3.58 \times 10^{3}$ & 39.14 & $5.05 \times 10^{2}$ & $101.06 \times 10^{4}$ & $3.87 \times 10^{-5}$ & $5.00 \times 10^{-4}$ & $1.97 \times 10^{-1}$ \\
\hline
\end{tabular}

Table A.12. $Z=10^{-4} \times Z_{\odot} 4 \mathrm{kpc}$.

\begin{tabular}{cccccccccc}
\hline \hline $\begin{array}{c}\text { Number } \\
\text { of SNe }\end{array}$ & $M_{* \mathrm{Fe}_{56}}$ & $M_{* \mathrm{O}_{16}}$ & $M_{* \mathrm{ej}}$ & $M_{\text {ShellFe }_{56}}$ & $M_{\text {ShellO }_{16}}$ & $M_{\mathrm{tot}}$ & $X_{* \mathrm{Fe}_{56}}$ & $X_{* \mathrm{O}_{16}}$ & {$[\mathrm{O} / \mathrm{Fe}]$} \\
\hline 10 & 0.97 & 8.58 & $1.16 \times 10^{2}$ & 1.09 & 9.55 & $100.71 \times 10^{4}$ & $1.08 \times 10^{-6}$ & $9.48 \times 10^{-6}$ & $2.89 \times 10^{-2}$ \\
50 & 4.26 & 42.06 & $5.20 \times 10^{2}$ & 4.38 & 43.03 & $100.75 \times 10^{4}$ & $4.35 \times 10^{-6}$ & $4.27 \times 10^{-5}$ & $7.86 \times 10^{-2}$ \\
100 & 7.79 & 82.80 & $9.42 \times 10^{2}$ & 7.91 & 83.77 & $100.79 \times 10^{4}$ & $7.85 \times 10^{-6}$ & $8.31 \times 10^{-5}$ & $1.11 \times 10^{-1}$ \\
500 & 27.36 & 390.33 & $3.58 \times 10^{3}$ & 27.47 & 391.30 & $101.05 \times 10^{4}$ & $2.71 \times 10^{-5}$ & $3.87 \times 10^{-4}$ & $2.40 \times 10^{-1}$ \\
\hline
\end{tabular}

